COMMUNICATIONS IN

ANALYSIS AND GEOMETRY

Volume 12, Number 3, 631-670, 2004

\title{
Rigidity of CR-immersions into Spheres
}

\author{
Peter Ebenfelt, Xiaojun Huang, and Dmitri Zaitsev
}

We consider local CR-immersions of a strictly pseudoconvex real hypersurface $M \subset \mathbb{C}^{n+1}$, near a point $p \in M$, into the unit sphere $\mathbb{S} \subset \mathbb{C}^{n+d+1}$ with $d>0$. Our main result is that if there is such an immersion $f:(M, p) \rightarrow \mathbb{S}$ and $d<n / 2$, then $f$ is rigid in the sense that any other immersion of $(M, p)$ into $\mathbb{S}$ is of the form $\varphi \circ f$, where $\varphi$ is a biholomorphic automorphism of the unit ball $\mathbb{B} \subset \mathbb{C}^{n+d+1}$. As an application of this result, we show that an isolated singulary of an irreducible analytic variety of codimension $d$ in $\mathbb{C}^{n+d+1}$ is uniquely determined up to affine linear transformations by the local CR geometry at a point of its Milnor link.

\section{Introduction.}

Let $X$ be a complex-analytic $(n+1)$-dimensional (not necessarily closed) irreducible variety in $\mathbb{C}^{n+d+1}$ and $\mathbb{S}_{\varepsilon}$ a real hypersphere of radius $\varepsilon>0$ such that the open ball $\mathbb{B}_{\varepsilon}$ (whose boundary is $\mathbb{S}_{\varepsilon}$ ) as well as $\mathbb{S}_{\varepsilon}$ intersect $X$; for convenience, we shall assume that the ball $\mathbb{B}_{\varepsilon}$ is centered at 0 . The intersection $K_{\varepsilon}:=X \cap \mathbb{S}_{\varepsilon}$ is then a real-analytic variety (whose set of regular points is a real hypersurface) in $X$. Let us denote by $M_{\varepsilon} \subset K_{\varepsilon}$ the relatively open subset of points where $X$ is nonsingular and meets the sphere transversally. For instance, if $X$ has an isolated singularity at $0 \in X$, then, for sufficiently small generic $\varepsilon>0, M_{\varepsilon}$ is all of $K_{\varepsilon}$; in this case, $M_{\varepsilon}$ is sometimes called the link of the singularity $(X, 0)$. In what follows, we shall refer to $M_{\varepsilon}$ as the $\mathbb{S}_{\mathcal{\varepsilon}}$-link (or simply the link) of the variety $X$, even though we do not necessarily assume that $\varepsilon$ is small or that $X$ has an isolated singularity at 0 (or even that the central point 0 is on $X$ ).

\footnotetext{
${ }^{1}$ The first author is a Royal Swedish Academy of Sciences Research Fellow supported in part by a grant from the Knut and Alice Wallenberg Foundation and by DMS-0100110.

${ }^{2}$ The second author is supported in part by DMS-0200689 and a grant from the Rutgers Research Council.

${ }^{3}$ The third author is supported in part by a grant from the Italian Consiglio Nazionale delle Ricerche.
} 
If $X^{\prime}$ is another variety of dimension $n+1$ in $\mathbb{C}^{n+d+1}$ and $M_{\varepsilon}^{\prime}$ is the link of $X^{\prime}$, and if there is a biholomorphic automorphism of the ball $\mathbb{B}_{\varepsilon}$ which sends the variety $X$ to $X^{\prime}$, then clearly the links $M_{\varepsilon}$ and $M_{\varepsilon}^{\prime}$ are CR-equivalent submanifolds of $\mathbb{S}_{\varepsilon}$; we should point out here that the automorphisms of the ball $\mathbb{B}_{\varepsilon}$ are linear fractional transformations of $\mathbb{C}^{n+d+1}$, which yield, by restriction, all the $\mathrm{CR}$ automorphisms of the sphere $\mathbb{S}_{\varepsilon}$. One may ask the converse:

(Q) Does the local CR-structure of the manifold $M_{\varepsilon}$ determine the variety $X$ uniquely (up to biholomorphic automorphisms of $\mathbb{B}_{\varepsilon}$ )?

That is, if $X^{\prime}$ is another irreducible analytic variety of the same dimension such that the $\mathbb{S}_{\varepsilon}$-link of $X^{\prime}$ is locally CR-equivalent to that of $X$, must then the varieties $X$ and $X^{\prime}$ be equivalent by a biholomorphic automorphism of the ball $\mathbb{B}_{\varepsilon}$ ? The answer is in general "no" as can be seen by the following well known example.

Example 1.1. Consider the so-called Whitney map $W: \mathbb{C}^{n+1} \rightarrow \mathbb{C}^{2 n+1}$,

$$
W\left(z_{1}, \ldots, z_{n+1}\right):=\left(z_{1}, \ldots, z_{n}, z_{1} z_{n+1}, z_{2} z_{n+1}, \ldots, z_{n} z_{n+1}, z_{n+1}^{2}\right),
$$

which is finite and hence the image is an irreducible analytic variety $X \subset \mathbb{C}^{2 n+1}$ (of codimension $d=n$ ). The variety $X$ is singular along the the complex line $z_{1}=\cdots=z_{n}=w_{1}=\ldots=w_{n}=0$, where $\left(z_{1}, \ldots, z_{n}, w_{1}, \ldots, w_{n+1}\right)$ denote the coordinates in $\mathbb{C}^{2 n+1}$. It is well known and easy to check that $W$ maps the unit sphere in $\mathbb{C}^{n+1}$ into the unit sphere in $\mathbb{C}^{2 n+1}$. Consequently, the link $M_{1}$ of $X$ is locally spherical (i.e. locally CR-equivalent to the sphere), and hence locally equivalent to $M_{1}^{\prime}$, where $M_{1}^{\prime}$ is the intersection of the unit sphere with $(n+1)$-plane $X^{\prime}=\left\{\left(z_{1}, \ldots, z_{n}, w_{1}, \ldots, w_{n+1}\right): z_{1}=\ldots=z_{n}=0\right\}$. However, the singular variety $X$ is clearly not biholomorphically equivalent (in any neighborhood of 0 ) to the nonsingular variety $X^{\prime}$.

On the other hand, it follows from the work of Webster [W79], Faran [Fa86], Cima-Suffridge [CS83] that if the link of $X$ is locally spherical and if the codimension $d$ is strictly less than $n$ (to exclude the situation in Example 1.1 above) then $X$ must be an $(n+1)$-plane. This answers in the affirmative the question (Q) above in this special case. See also the work of Cima-Suffridge [CS90], Forstneric [F89], and the second author [Hu99] in some other related directions.

In this paper, we shall consider the general form of the question (Q). We shall prove that if the codimension of the variety $X$ in $\mathbb{C}^{n+d+1}$ is less than half 
its dimension minus one, then the answer to (Q) is affirmative. More generally, we consider the situation where $M_{\varepsilon}=M$ is a smooth (meaning here always $\mathcal{C}^{\infty}$ ) abstract CR-manifold of hypersurface type (a CR-hypersurface) and prove the following:

Theorem 1.2. Let $M$ be a connected smooth CR-hypersurface of dimension $2 n+1$. If $d<n / 2$, then any smooth $C R$-immersion $f$ of $M$ into the unit sphere $\mathbb{S}$ in $\mathbb{C}^{n+d+1}$ is rigid. That is, any other smooth CR-immersion $\tilde{f}: M \rightarrow \mathbb{S}$ is related to $f$ by $\tilde{f}=\varphi \circ f$, where $\varphi$ is a $C R$-automorphism of $\mathbb{S}$.

In the case $d=1$, the conclusion in Theorem 1.2 follows from the work of Webster [W79].

Theorem 1.2 can be directly applied to study the question (Q) above. Let us first consider the case where $X$ has an isolated singularity at $0 \in X$ and no other singularity of $X$ is inside the ball $\mathbb{B}_{\varepsilon}$. If $X^{\prime}$ is another such variety and if their links $M_{\varepsilon}, M_{\varepsilon}^{\prime}$ are locally $\mathrm{CR}$ equivalent at $p \in M_{\varepsilon}$ and $p^{\prime} \in M_{\varepsilon}^{\prime}$, then in view of Theorem 1.2 (the unit sphere in that theorem can of course be replaced by a sphere of any radius) there is a biholomorphic automorphism $\varphi$ of $\mathbb{B}_{\varepsilon}$ (extending as a birational transformation of the whole space $\mathbb{C}^{n+d+1}$ ) which sends an open piece of $M_{\varepsilon}$ to an open piece of $M_{\varepsilon}^{\prime}$. If we, in addition, assume that $X \cap \mathbb{B}_{\varepsilon}$ and $X^{\prime} \cap \mathbb{B}_{\varepsilon}$ are connected, then we conclude that $\varphi$ sends $X \cap \mathbb{B}_{\varepsilon}$ to $X^{\prime} \cap \mathbb{B}_{\varepsilon}$. Since the only singularities of $X$ and $X^{\prime}$ inside $\mathbb{B}_{\varepsilon}$ are at $0, \varphi$ must also send 0 to 0 . By also using the fact that the only biholomorphic automorphisms of the ball $\mathbb{B}_{\varepsilon}$ which fix the origin are unitary linear transformation of $\mathbb{C}^{n+d+1}$, we obtain the following:

Corollary 1.3. Let $X, X^{\prime} \subset \mathbb{C}^{n+d+1}$ be irreducible complex analytic varieties of codimension $d$ through 0 . Let $M_{\varepsilon}, M_{\varepsilon}^{\prime}$ denote their manifolds of regular intersection with a sphere $\mathbb{S}_{\varepsilon}$ of radius $\varepsilon>0$ centered at $0 \in \mathbb{C}^{n+d+1}$. Assume that both $X$ and $X^{\prime}$ have isolated singularities at 0 , no other singular points in $\mathbb{B}_{\varepsilon}$, and $X \cap \mathbb{B}_{\varepsilon}, X^{\prime} \cap \mathbb{B}_{\varepsilon}$ are connected. If $d<n / 2$ and $M_{\varepsilon}$ and $M_{\varepsilon}^{\prime}$ are locally $C R$-equivalent at some points $q \in M_{\varepsilon}$ and $q^{\prime} \in M_{\varepsilon}^{\prime}$, then there exists a unitary linear transformation which maps $X \cap \mathbb{B}_{\varepsilon}$ to $X^{\prime} \cap \mathbb{B}_{\varepsilon}^{\prime}$.

We can also consider the general case where $X$ and $X^{\prime}$ are not assumed to have isolated singularities (and 0 is not necessarily a point on $X \cap X^{\prime}$ ). A similar argument to the one above yields the following result:

Corollary 1.4. Let $X, X^{\prime} \subset \mathbb{C}^{n+d+1}$ be irreducible complex analytic varieties of codimension $d$ and let $M_{\varepsilon}, M_{\varepsilon}^{\prime}$ denote their manifolds of regular 
intersection with a sphere $\mathbb{S}_{\varepsilon}$ of radius $\varepsilon>0$ centered at $0 \in \mathbb{C}^{n+d+1}$. If $d<n / 2$ and $M_{\varepsilon}$ and $M_{\varepsilon}^{\prime}$ are locally $C R$-equivalent at some points $q \in M_{\varepsilon}$ and $q^{\prime} \in M_{\varepsilon}^{\prime}$, then there exists a biholomorphic automorphism of the ball $\mathbb{B}_{\varepsilon}$ whose birational extension to $\mathbb{C}^{n+d+1}$ sends $(X, q)$ to $\left(X^{\prime}, q^{\prime}\right)$ (and $X$ into a subvariety containing $X^{\prime}$ as an open subset).

In a different direction, we also obtain the following result as a direct consequence of Theorem 1.2; the simple argument needed to derive it from Theorem 1.2 is left to the reader.

Corollary 1.5. Let $D$ be a connected relatively compact open subset of a complex manifold and assume that $D$ has a smooth strictly pseudoconvex boundary $\partial D$. Assume that there is a proper embedding of $D$ into the unit ball $\mathbb{B}_{1} \subset \mathbb{C}^{n+d+1}$, with $d<n / 2$, which extends smoothly to the boundary $\partial D$. If $g$ is a local CR-diffeomorphism sending a relatively open piece of $\partial D$ into $\partial D$, then $g$ extends as a biholomorphic automorphism of $D$.

Theorem 1.2 above will follow from the more general Theorem 2.1 below, where a higher codimension $d$ is allowed provided that $M_{\varepsilon}$ is suitably degenerate. As a corollary, we recover the result about $(n+1)$-planes mentioned above.

The proof of Theorem 2.1 will be completed in $\S 9$. It decomposes naturally into two parts. The first part consists of showing that if the mapping is degenerate (in a certain sense to be defined in the next section), then the image $f(M)$ is in fact contained in the intersection of the sphere with a lower dimensional complex plane (see Theorem 2.2). Using this fact, we reduce the proof to the case where $f$ is finitely nondegenerate (see $\S 2$ ). The reader is referred to $\S 2$ for the statements of mentioned results and a more detailed outline of the proof of Theorem 2.1.

\section{Further results.}

Let $M$ be CR-hypersurface of dimension $2 n+1$ and denote by $\mathcal{V}=T^{0,1} M \subset$ $\mathbb{C} T M$ its CR-bundle; the reader is referred e.g. to [BER99] for basic notions and facts about CR-manifolds (see also $\S 3$ ). Recall that a mapping $f=$ $\left(f_{1}, \ldots, f_{k}\right): M \rightarrow \mathbb{C}^{k}$ is called $\mathrm{CR}$ if $f_{*}\left(\mathcal{V}_{p}\right) \subset T_{f(p)}^{0,1} \mathbb{C}^{k}$ for every $p \in M$. This is equivalent to saying that $L f_{j}=0$ for every $j=1, \ldots k$ and every $(0,1)$ vector field $L$ (i.e. a section in $\mathcal{V}$ ).

An important step in proving Theorem 1.2 will be to classify possible CR-immersions according to their degeneracy. Let $\hat{M} \subset \mathbb{C}^{\hat{n}+1}$ be a real hypersurface (and hence also a CR-manifold) and $f: M \rightarrow \mathbb{C}^{\hat{n}+1}$ be a $\mathrm{CR}$ 
mapping sending $M$ into $\hat{M}$. We shall refer to $d=\hat{n}-n$ as the codimension of the mapping $f$; thus, $\hat{M}$ is a real hypersurface in $\mathbb{C}^{n+d+1}$. Let $p$ be a point in $M$ and $\hat{\rho}$ a local defining function for $\hat{M}$ near $\hat{p}:=f(p) \in \hat{M}$. Following Lamel [L01a], define an increasing sequence of subspaces $E_{k}(p)$ of the space $T_{\hat{p}}^{\prime} \mathbb{C}^{n+d+1}$ of $(1,0)$ covectors as follows. Let $L_{\overline{1}}, \ldots, L_{\bar{n}}$ be a basis of $(0,1)$ vector fields on $M$ near $p$ and define

$$
E_{k}(p):=\operatorname{span}_{\mathbb{C}}\left\{L^{\bar{J}}\left(\hat{\rho}_{Z^{\prime}} \circ f\right)(p): J \in\left(\mathbb{Z}_{+}\right)^{n}, 0 \leq|J| \leq k\right\} \subset T_{\hat{p}}^{\prime} \mathbb{C}^{n+d+1},
$$

where $\hat{\rho}_{Z^{\prime}}=\partial \hat{\rho}$ is represented by vectors in $\mathbb{C}^{n+d+1}$ in some local coordinate system $Z^{\prime}$ near $\hat{p}$; we use here standard multi-index notation $L^{\bar{J}}:=L_{\overline{1}}^{\bar{J}_{1}} \ldots L_{\bar{n}}^{\bar{J}_{n}}$ and $|J|:=J_{1}+\ldots+J_{n}$. One can show (cf. [L01a]) that $E_{k}(p)$ is independent of the choice of local defining function $\hat{\rho}$ and coordinates $Z^{\prime}$, as well as of the choice of basis of the CR vector fields $L_{\overline{1}}, \ldots, L_{\bar{n}}$. We shall say, again following Lamel [L01a, L01b] that $f:(M, p) \rightarrow \hat{M}$ is $\left(k_{0}, s_{0}\right)$-degenerate at $p$ if $s_{0}$ is the minimum of the decreasing sequence of codimensions of $E_{k}(p)$ in $\mathbb{C}^{n+d+1}$, i.e.

$$
s_{0}=s(p):=\min _{k} \operatorname{codim} E_{k}(p)
$$

and $k=k_{0}$ is the smallest integer for which this minimum is attained. If $E_{k}(p)=T_{\hat{p}}^{\prime} \mathbb{C}^{n+d+1}$ for some $k$, i.e. if $f$ is $\left(k_{0}, 0\right)$-degenerate at $p$ for some $k_{0}$, it is said to be $k_{0}$-nondegenerate (at $p$ ) or finitely nondegenerate without specifying $k_{0}$.

Let us call the degeneracy of $f$ the minimum of $s(p)$ as defined in (2.2) for $p \in M$. We have the following result which, in view of Theorem 7.3 and Proposition 7.1 below, implies Theorem 1.2.

Theorem 2.1. Let $f: M \rightarrow \mathbb{S}$ be a smooth CR-immersion of a smooth connected CR-hypersurface $M$ of dimension $2 n+1$ into the unit sphere $\mathbb{S}$ in $\mathbb{C}^{n+d+1}$ and denote by $s$ be the degeneracy of $f$. If $d-s<n / 2$, then $f$ is rigid among smooth $C R$-immersions having the same degeneracy. That is, any other such CR-immersion $\tilde{f}: M \rightarrow \mathbb{S}$ is related to $f$ by $\tilde{f}=\varphi \circ f$, where $\varphi$ is a $C R$-automorphism of $\mathbb{S}$.

It is not difficult to see from the definition that, if $f(M)$ is contained in a complex plane of codimension $s$ in $\mathbb{C}^{n+d+1}$, then the degeneracy of $f$ is at least $s$. A important ingredient in the proof of Theorem 2.1 is the following (partial) converse, which also seems to be of independent interest. 
Theorem 2.2. Let $f: M \rightarrow \mathbb{S}$ be a smooth CR-immersion of a smooth connected CR-hypersurface $M$ of dimension $2 n+1$ into the unit sphere $\mathbb{S}$ in $\mathbb{C}^{n+d+1}$. Let $s$ be the degeneracy of $f$. If $d-s<n$, then $f(M)$ is contained in the intersection of $\mathbb{S}$ with a complex plane $P \subset \mathbb{C}^{n+d+1}$ of codimension $s$. Moreover, if $f: M \rightarrow \mathbb{S}$ is $\left(k_{0}, s\right)$-nondegenerate at a point $p \in M$, then it is $k_{0}$-nondegenerate there as a mapping $f: M \rightarrow \mathbb{S} \cap P$.

Another important ingredient in the proof of Theorem 1.2 will be the $\mathrm{CR}$ analogue of the classical Gauss equation whic, as the reader may recall, relates the Riemannian curvature tensors of a manifold and its submanifold with the second fundamental form composed with the Riemannian metric (see e.g. [KN69]). The (extrinsic) second fundamental form for a CR-mapping $f: M \rightarrow \hat{M}$ between real hypersurfaces $M \subset \mathbb{C}^{n+1}$ and $\hat{M} \subset \mathbb{C}^{n+d+1}$ can be defined (up to a scalar factor) by

$$
\Pi\left(X_{p}, Y_{p}\right):=\overline{\pi\left(X Y\left(\hat{\rho}_{\bar{Z}^{\prime}} \circ f\right)(p)\right)} \in \overline{T_{\hat{p}}^{\prime} \hat{M} / E_{1}(p)},
$$

where $\pi: T_{\hat{p}}^{\prime} \hat{M} \rightarrow T_{\hat{p}}^{\prime} \hat{M} / E_{1}(p)$ is the projection and $X, Y$ are any $(1,0)$ vector fields on $M$ extending given vectors $X_{p}, Y_{p} \in T_{p}^{1,0} M$. In the case where $\hat{M}$ (and hence also $M$ ) is strictly pseudoconvex, the Levi form of $\hat{M}$ (at $\hat{p}$ ) with respect to $\hat{\rho}$ defines an isomorphism $\overline{T_{\hat{p}}^{\prime} \hat{M} / E_{1}(p)} \cong T_{\hat{p}}^{1,0} \hat{M} / f_{*} T_{p}^{1,0} M$ and hence the second fundamental form can be viewed as an $\mathbb{C}$-bilinear symmetric form

$$
\Pi_{p}: T_{p}^{1,0} M \times T_{p}^{1,0} M \rightarrow T_{\hat{p}}^{1,0} \hat{M} / f_{*} T_{p}^{1,0} M
$$

that does not depend anymore on the choice of $\hat{\rho}$. In $\S 4$ (see equation (4.7)), we shall show that $\Pi$ is indeed the second fundamental form (in the classical sense) of $f$ with respect to Webster's pseudohermitian connection induced by the CR-structure (and a choice of contact form). We shall say that the second fundamental form of $f$ is nondegenerate at $p$ if its values span the target space. This is easily seen to be equivalent to $E_{2}(p)=T_{\hat{p}}^{\prime} \mathbb{C}^{n+d+1}$ or, in the notation above, to $f$ being 2-nondegenerate at $p$.

The CR analogue of the Weyl curvature tensor in Riemannian geometry is given by the tangential pseudoconformal curvature tensor

$$
S: T_{p}^{1,0} M \times T_{p}^{1,0} M \times T_{p}^{1,0} M \times T_{p}^{1,0} M \rightarrow \mathbb{C} T_{p} M /\left(T_{p}^{1,0} M \oplus T_{p}^{0,1} M\right)
$$

defined by Chern and Moser [CM74] for every Levi-nondegenerate CRhypersurface $M$ (in fact, it is defined there as a tensor on a principal bundle over $M$ which can be pulled back to $M$ as will be explained in $\S 3$, see also 
[W79]). The role of the Riemannian metric itself is played by the Levi form which can be invariantly seen as a Hermitian bilinear map

$$
L: T_{p}^{1,0} M \times T_{p}^{1,0} M \rightarrow \mathbb{C} T_{p} M /\left(T_{p}^{1,0} M \oplus T_{p}^{0,1} M\right) .
$$

In the following we identify the quotient $T_{\hat{p}}^{1,0} \hat{M} / f_{*} T_{p}^{1,0} M$ with the orthogonal complement of $f_{*} T_{p}^{1,0} M$ in $T_{\hat{p}}^{1,0} \hat{M}$ with respect to the Levi form of $\hat{M}$. We shall also use the notation $f_{*}$ for the mapping $\mathbb{C} T_{p} M /\left(T_{p}^{1,0} M \oplus T_{p}^{0,1} M\right) \rightarrow$ $\mathbb{C} \hat{T}_{\hat{p}} M /\left(T_{\hat{p}}^{1,0} \hat{M} \oplus T_{\hat{p}}^{0,1} \hat{M}\right)$ induced by $f_{*}: \mathbb{C} T_{p} M \rightarrow \mathbb{C} T_{\hat{p}} \hat{M}$. The $\mathrm{CR}$ analogue of the Gauss identity mentioned above can be now stated as follows.

Theorem 2.3. Let $f: M \rightarrow \hat{M}$ be a smooth CR-immersion of a smooth connected CR-hypersurface $M$ into a strongly pseudoconvex CRhypersurface $\hat{M}$. Denote by $\Pi$ the second fundamental form of $f$, by $L$ and $S$ the Levi form and the tangential pseudoconformal curvature tensor for $M$ and by $\hat{L}$ and $\hat{S}$ the corresponding tensors for $\hat{M}$. Then there exists a Hermitian form $H: T_{p}^{1,0} M \times T_{p}^{1,0} M \rightarrow \mathbb{C}$ such that the identity

$$
\begin{aligned}
\hat{S}\left(f_{*} V, f_{*} V, f_{*} V, f_{*} V\right)-f_{*} S(V, V, V, V)=\hat{L}( & \Pi(V, V), \Pi(V, V)) \\
& +f_{*} L(V, V) H(V, V)
\end{aligned}
$$

holds for every $V \in T_{p}^{1,0} M$.

The reader is referred to $\S 5$ for more details on this Gauss equation and to Proposition 5.2 from which it immediately follows. Here we only briefly mention that the term involving $H$ on the right hand side (which will be called conformally equivalent to 0 , cf. §3) can be expressed from (2.5) in terms of $\hat{S}, \hat{L}, L$ and $\Pi$ and hence (2.5) can be given a more explicit form (see $(5.8))$.

We conclude this section by giving an outline of the paper and proofs of Theorems 2.2 and 2.1. In $\S 3$ we recall the construction of the pseudohermitian connection defined by Webster [W78] on a given strongly pseudoconvex CR-hypersurface with a fixed contact form and show the relation to the pseudoconformal connection defined by Chern and Moser [CM74] in terms of the forms (3.11). In $\S 4$ we show the existence of coframes suitably adapted to a pair $(M, \hat{M})$ of strongly pseudoconvex CR-hypersurfaces, where $M$ is a submanifold of $\hat{M}$. It is further shown the relation of the pseudohermitian connection with respect to such a coframe with the second fundamental form as defined above. $\S 5$ is devoted to the proof of pseudohermitian and pseudoconformal analogues of the Gauss equation (Propositions 5.1 and 5.2 
from which Theorem 2.3 follows). As one of the main consequences (see Corollary 5.5(ii)) we obtain the following, which seems interesting in its own right:

Under the assumptions of Theorem 1.2, the second fundamental form $\Pi$ at a point $p \in M$ is uniquely determined, up to a unitary transformation of the target space, by the tangential pseudoconformal curvature tensor $S$ of $M$ at $p$ (and, hence, does not depend on $f$ ).

We also show (see Corollary 5.5(i)) that if $d<n$ and $M$ is the sphere, then the second fundamental form of $f$ vanishes identically. Combining the latter result with Theorem 2.1 above ( $s=d$ in this case), we recover the result of Faran et al mentioned above, that any CR-immersion of the sphere in $\mathbb{C}^{n+1}$ into the sphere in $\mathbb{C}^{n+d+1}$, with $d<n$, is equivalent (after composing to the left and right with automorphisms of the spheres) to the linear embedding.

In $\S 6$ we express the forms defining the pseudoconformal connection of $\hat{M}$ pulled back to $M$ in terms of the corresponding forms for $M$ and the second fundamental form $\Pi$ of $f$ under the additional assumption that $\Pi$ is nondegenerate. If it is not, higher order covariant derivatives of $\Pi$ are needed; this is dealt with in $\S 7$. We begin the latter section by showing how covariant derivatives of $\Pi$ can be used to determine the spaces $E_{k}$ defined in (2.1) (see Proposition 7.1). We then prove (Theorem 7.2) that, if $f$ is $k_{0^{-}}$ nondegenerate, then the pseudonformal connection of $\hat{M}$ pulled back to $M$ is uniquely determined by the covariant derivatives of $\Pi$ up to order $k_{0}-1$. The second main result in $\S 7$ (Theorem 7.3) then states that equality of the latter derivatives for two immersions always holds, after possibly a unitary change of adapted coframes, provided that the codimension $d<n / 2$. An important technical point here is to obtain a commuting relation between the covariant derivatives (Lemma 7.4). Finally, in $\S 8$ we recall from [CM74] how adapted $Q$-frames on a sphere and their Maurer-Cartan forms are related to the pseudoconformal connection forms (see (8.5)). We then complete the proof of Theorem 2.1 in the case $s=0$, i.e. when $f$ is finitely nondegenerate at some point. In this case the results of $\S 7$ yield that the covariant derivatives of $\Pi$ and hence the pulled back pseudoconformal connection does not depend on $f$. From (8.5) we further conclude that also Maurer-Cartan forms for associated adapted $Q$-frames do not depend on $f$ and the proof is completed as in [W79] by general ODE arguments. $\S 9$ is mainly devoted to the proof of Theorem 2.2 which is obtained as a consequence of the fact that the span of certain vectors in a suitable adapted $Q$-frame is independent of the reference point. Theorem 2.2 is then used to complete the proof of Theorem 2.1 by reducing the general case to the case $s=0$ treated in $\S 8$. 


\section{Preliminaries.}

Let $M$ be a strictly pseudoconvex CR-manifold (which in this paper will always be understood to be of hypersurface type) of dimension $2 n+1$. We shall write

$$
\begin{aligned}
& T^{c} M:=T M \cap i T M \subset T M, \\
& \mathcal{V}=T^{0,1} M:=\left\{X+i J X: X \in T^{c} M\right\} \subset \mathbb{C} T M:=T M \otimes \mathbb{C}
\end{aligned}
$$

for its maximal complex tangent bundle and CR-bundle respectively which are both complex rank $n$ bundles. Here $J: T^{c} M \rightarrow T^{c} M$ is the complex structure. We also consider the cotangent bundles

$$
T^{0} M:=(\mathcal{V} \oplus \overline{\mathcal{V}})^{\perp}, T^{\prime} M:=\mathcal{V}^{\perp} .
$$

Thus, $T^{0} M$ and $T^{\prime} M$ are rank one and rank $n+1$ subbundles of $\mathbb{C} T^{*} M$ respectively with $T^{0} \subset T^{\prime} M$. The bundle $T^{\prime} M$ is called the holomorphic or $(1,0)$ cotangent bundle of $M$. As usual, a section of $\Lambda^{p}\left(T^{\prime} M\right)$ is called a $(p, 0)$-form on $M$. A real nonvanishing section $\theta$ of $T^{0} M$ is called a contact form. A choice of a contact form defines uniquely a real vector field $T$, the characteristic (or Reeb) vector field of $\theta$ (cf. e.g. [Ho98]), by

$$
T\lrcorner d \theta=0,\langle\theta, T\rangle=1,
$$

where $\lrcorner$ denotes contraction (or interior multiplication). Indeed, since $d \theta$ is a degenerate 2 -form on $T M$ but nondegenerate on the real hyperplanes defined by $\theta=0$ in $T M$, one can always find $T$ satisfying (3.2) in the kernel of $d \theta$.

We will follow the notation of [CM74] and [W78], in particular, we use the summation convention and small Greek indices will always run over the set $\{1, \ldots, n\}$. A typical tensor will be written as $S_{\alpha}{ }_{\mu \bar{\nu}}$, where an index without (resp. with) conjugation indicates $\mathbb{C}$-linear (resp. $\mathbb{C}$-antilinear) dependence in the corresponding argument. Here such a tensor $S_{\alpha}{ }_{\mu \bar{\nu}}$ can be considered as an $\mathbb{R}$-multilinear complex-valued function on $\mathcal{V} \times \mathcal{V}^{*} \times \mathcal{V} \times \mathcal{V}$. The tensors will not be necessarily symmetric in their indices and hence the order of the indices will be important and will be explicitly indicated. Simultaneous conjugation of all indices corresponds to conjugation of the tensor: $S_{\bar{\alpha}}{ }^{\bar{\beta}}{ }_{\mu}=\overline{S_{\alpha}{ }^{\beta} \bar{\nu}}$. On the other hand, there is no a priori relation e.g. between $S_{\alpha}{ }^{\beta} \mu \bar{\nu}$ and $S_{\alpha}{ }^{\beta} \mu \nu$. The Levi form matrix $\left(g_{\alpha \bar{\beta}}\right)$ of $M$ (relative to

a given contact form) and its inverse $\left(g^{\alpha \bar{\beta}}\right)$ will be used to raise and lower indices (without changing their order): $S_{\alpha \bar{\beta}} \mu \bar{\nu}=g_{\gamma \bar{\beta}} S_{\alpha}{ }_{\mu \bar{\nu}}$. More generally, 
the same notation will be used for indexed functions on $M$ that may not necessarily transform as tensors, e.g. for connection matrices etc.

If we choose a basis $L_{\alpha}, \alpha=1, \ldots, n$, of $(1,0)$ vector fields (i.e. sections of $\left.T^{1,0} M=\overline{\mathcal{V}}\right)$, so that $\left(T, L_{\alpha}, L_{\bar{\alpha}}\right)$ is a frame for $\mathbb{C} T M$, then the first equation in (3.2) is equivalent to

$$
d \theta=i g_{\alpha \bar{\beta}} \theta^{\alpha} \wedge \theta^{\bar{\beta}}
$$

$\left(g_{\alpha \bar{\beta}}\right)$ is the (Hermitian) Levi form matrix as above and $\left(\theta, \theta^{\alpha}, \theta^{\bar{\alpha}}\right)$ is the coframe (i.e. a collection of linearly independent 1-forms spanning $\mathbb{C} T^{*} M$ ) dual to $\left(T, L_{\alpha}, L_{\bar{\alpha}}\right)$ (for brevity, we shall say that $\left(\theta, \theta^{\alpha}\right)$ is the coframe dual to $\left.\left(T, L_{\alpha}\right)\right)$. Note that $\theta$ and $T$ are real whereas $\theta^{\alpha}$ and $L_{\alpha}$ always have nontrivial real and imaginary parts.

Following Webster [W78], we call a coframe $\left(\theta, \theta^{\alpha}\right)$ (and its dual frame $\left(T, L_{\alpha}\right)$ ), where $\theta$ is a contact form, admissible if (3.3) holds or, equivalently, if $T$ is characteristic for $\theta$ in the sense of (3.2). Observe that (by the uniqueness of the Reeb vector field) for a given contact form $\theta$ on $M$, the admissible coframes are determined up to transformations

$$
\tilde{\theta}^{\alpha}=u_{\beta}{ }^{\alpha} \theta^{\beta}, \quad\left(u_{\beta}^{\alpha}\right) \in \mathrm{GL}\left(\mathbb{C}^{n}\right) .
$$

Every choice of a contact form $\theta$ on $M$ is called pseudohermitian structure and defines a Hermitian metric on $\mathcal{V}$ (and on $\overline{\mathcal{V}}$ ) via the (positive-definite) Levi form. For every such $\theta$, Tanaka [T75] and Webster [W78] defined a pseudohermitian connection $\nabla$ on $\overline{\mathcal{V}}$ (and also on $\mathbb{C} T M$ ) which is expressed relative to an admissible coframe $\left(\theta, \theta^{\alpha}\right)$ by

$$
\nabla L_{\alpha}:=\omega_{\alpha}^{\beta} \otimes L_{\beta},
$$

where the 1-forms $\omega_{\alpha}^{\beta}$ on $M$ are uniquely determined by the conditions

$$
\begin{aligned}
d \theta^{\beta} & =\theta^{\alpha} \wedge \omega_{\alpha}{ }^{\beta} \quad \bmod \theta \wedge \theta^{\bar{\alpha}}, \\
d g_{\alpha \bar{\beta}} & =\omega_{\alpha \bar{\beta}}+\omega_{\bar{\beta} \alpha} .
\end{aligned}
$$

The first condition in (3.6) can be rewritten as

$$
d \theta^{\beta}=\theta^{\alpha} \wedge \omega_{\alpha}^{\beta}+\theta \wedge \tau^{\beta}, \quad \tau^{\beta}=A^{\beta} \theta^{\bar{\nu}}, \quad A^{\alpha \beta}=A^{\beta \alpha}
$$

for suitable uniquely determined torsion matrix $\left(A^{\beta} \bar{\alpha}\right)$, where the last symmetry relation holds automatically (see [W78]). (More precisely, the forms $\omega_{\alpha}{ }^{\beta}$ and $\tau^{\beta}$ are first defined in [W78] on the principal bundle $P$ of all admissible coframes $\left(\theta, \theta^{\alpha}\right)$ on $M$ with fixed $\theta$ and are then pulled back to $M$ via a section of $P$ corresponding to a choice of such a coframe.) 
The curvature of the pseudohermitian connection is given, in view of [W78, (1.27), (1.41)], by

$d \omega_{\alpha}{ }^{\beta}-\omega_{\alpha}{ }^{\gamma} \wedge \omega_{\gamma}{ }^{\beta}=R_{\alpha}{ }^{\beta}{ }_{\mu \bar{\nu}} \theta^{\mu} \wedge \theta^{\bar{\nu}}+W_{\alpha}{ }^{\beta}{ }_{\mu} \theta^{\mu} \wedge \theta-W^{\beta}{ }_{\alpha \bar{\nu}} \theta^{\bar{\nu}} \wedge \theta+i \theta_{\alpha} \wedge \tau^{\beta}-i \tau_{\alpha} \wedge \theta^{\beta}$,

where the functions $R_{\alpha}{ }^{\beta}{ }_{\nu \bar{\mu}}$ and $W_{\alpha}{ }^{\beta}{ }_{\nu}$ represent the pseudohermitian curvature of $(M, \theta)$. It has been noticed by Lee [Le88] that the components $W_{\alpha}{ }^{\beta} \mu$ can be in fact obtained as covariant derivatives of the torsion matrix $A^{\beta} \bar{\alpha}$ in (3.7). Here we denote the covariant differentiation operator with respect to the pseudohermitian connection $\nabla$ also by $\nabla$ and its components by indices preceded by a semicolumn, where the index 0 is used to denote the covariant derivative with respect to $T$; thus, e.g.

$$
\nabla A^{\beta} \bar{\alpha}_{\bar{\alpha}}=d A^{\beta} \bar{\alpha}+A^{\mu} \bar{\alpha}_{\omega_{\mu}}^{\beta}-A^{\beta}{ }_{\bar{\nu}} \omega_{\bar{\alpha}}{ }^{\bar{\nu}}=A^{\beta} \bar{\alpha} ; 0_{0} \theta+A^{\beta} \bar{\alpha} ; \nu \theta^{\nu}+A^{\beta} \bar{\alpha} ; \bar{\nu} \theta^{\bar{\nu}} .
$$

In this notation the above mentioned relation reads [Le88, (2.4)]:

$$
W_{\alpha}^{\beta}{ }_{\mu}=A_{\alpha \mu ;}^{\beta}, \quad W_{\alpha \bar{\nu}}^{\beta}=A_{\bar{\nu} ; \alpha}^{\beta} .
$$

We shall also need the Chern-Moser coframe bundle $Y$ over $M$. Recall $[\mathrm{CM} 74, \S 4]$ that $Y$ is the bundle of the coframes $\left(\omega, \omega^{\alpha}, \omega^{\bar{\alpha}}, \varphi\right)$ on the real line bundle $\pi_{E}: E \rightarrow M$ (of all contact forms) satisfying $d \omega=i g_{\alpha \bar{\beta}} \omega^{\alpha} \wedge$ $\omega^{\bar{\beta}}+\omega \wedge \varphi$, where $\omega^{\alpha}$ is in $\pi_{E}^{*}\left(T^{\prime} M\right)$ and $\omega$ is the canonical form on $E$ given by $\omega(\theta)(X):=\theta\left(\left(\pi_{E}\right)_{*} X\right)$ for $\theta \in E, X \in T_{\theta} E$. Similarly, canonical forms $\omega, \omega^{\alpha}, \omega^{\bar{\alpha}}, \varphi$ are defined on $Y$ (here the same letters are used as for the coframe by a slight abuse of notation). Chern and Moser [CM74] showed that these forms can be completed to a natural parallelism on $Y$ given by the coframe of 1 -forms

$$
\left(\omega, \omega^{\alpha}, \omega^{\bar{\alpha}}, \varphi, \varphi_{\beta}^{\alpha}, \varphi^{\alpha}, \varphi^{\bar{\alpha}}, \psi\right)
$$

defining the pseudoconformal connection on $Y$ and satisfying the structure 
equations (see [CM74] and its appendix)

$$
\begin{aligned}
& \varphi_{\alpha \bar{\beta}}+\varphi_{\bar{\beta} \alpha}=g_{\alpha \bar{\beta}} \varphi \\
& d \omega=i \omega^{\mu} \wedge \omega_{\mu}+\omega \wedge \varphi, \\
& d \omega^{\alpha}=\omega^{\mu} \wedge \varphi_{\mu}{ }^{\alpha}+\omega \wedge \varphi^{\alpha}, \\
& d \varphi=i \omega_{\bar{\nu}} \wedge \varphi^{\bar{\nu}}+i \varphi_{\bar{\nu}} \wedge \omega^{\bar{\nu}}+\omega \wedge \psi, \\
& d \varphi_{\beta}{ }^{\alpha}=\varphi_{\beta}{ }^{\mu} \wedge \varphi_{\mu}{ }^{\alpha}+i \omega_{\beta} \wedge \varphi^{\alpha}-i \varphi_{\beta} \wedge \omega^{\alpha}-i \delta_{\beta}{ }^{\alpha} \varphi_{\mu} \wedge \omega^{\mu}-\frac{\delta_{\beta}{ }^{\alpha}}{2} \psi \wedge \omega+\Phi_{\beta}{ }^{\alpha}, \\
& d \varphi^{\alpha}=\varphi \wedge \varphi^{\alpha}+\varphi^{\mu} \wedge \varphi_{\mu}{ }^{\alpha}-\frac{1}{2} \psi \wedge \omega^{\alpha}+\Phi^{\alpha}, \\
& d \psi=\varphi \wedge \psi+2 i \varphi^{\mu} \wedge \varphi_{\mu}+\Psi,
\end{aligned}
$$

where the curvature 2 -forms $\Phi_{\beta}^{\alpha}, \Phi^{\alpha}, \Psi$ can be decomposed as

$$
\begin{aligned}
& \Phi_{\beta}{ }^{\alpha}=S_{\beta}{ }^{\alpha}{ }_{\mu \bar{\nu}} \omega^{\mu} \wedge \omega^{\bar{\nu}}+V_{\beta}{ }^{\alpha}{ }_{\mu} \omega^{\mu} \wedge \omega+V^{\alpha}{ }_{\beta \bar{\nu}} \omega \wedge \omega^{\bar{\nu}}, \\
& \Phi^{\alpha}=V^{\alpha}{ }_{\mu \bar{\nu}} \omega^{\mu} \wedge \omega^{\bar{\nu}}+P_{\mu}{ }^{\alpha} \omega^{\mu} \wedge \omega+Q_{\bar{\nu}}{ }^{\alpha} \omega^{\bar{\nu}} \wedge \omega, \\
& \Psi=-2 i P_{\mu \bar{\nu}} \omega^{\mu} \wedge \omega^{\bar{\nu}}+R_{\mu} \omega^{\mu} \wedge \omega+R_{\bar{\nu}} \omega^{\bar{\nu}} \wedge \omega,
\end{aligned}
$$

where the functions $S_{\beta}{ }^{\alpha}{ }_{\mu \bar{\nu}}, V_{\beta}{ }^{\alpha}{ }_{\mu}, P_{\mu}{ }^{\alpha}, Q_{\bar{\nu}}{ }^{\alpha}, R_{\mu}$ together represent the pseudoconformal curvature of $M$ (the indices of $S_{\beta}{ }^{\alpha}{ }_{\mu \nu}$ here are interchanged comparing to [CM74] to make them consistent with indices of $R_{\beta}{ }^{\alpha}{ }_{\mu \nu}$ in (3.8)). As in [CM74] we restrict our attention here to coframes $\left(\theta, \theta^{\alpha}\right)$ for which the Levi form $\left(g_{\alpha \bar{\beta}}\right)$ is constant. The 1-forms $\varphi^{\alpha}, \varphi^{\bar{\alpha}}, \varphi_{\beta}{ }^{\alpha}, \psi$ are uniquely determined by requiring the coefficients in (3.13) to satisfy certain symmetry and trace conditions (see [CM74] and the appendix), e.g.

$$
S_{\alpha \bar{\beta} \mu \bar{\nu}}=S_{\mu \bar{\beta} \alpha \bar{\nu}}=S_{\mu \bar{\nu} \alpha \bar{\beta}}=S_{\bar{\nu} \mu \bar{\beta} \alpha}, \quad S_{\mu}{ }_{\alpha \bar{\beta}}=V_{\alpha}{ }^{\mu}{ }_{\mu}=P_{\mu}{ }^{\mu}=0 .
$$

Let us fix a contact form $\theta$ that defines a section $M \rightarrow E$. Then any admissible coframe $\left(\theta, \theta^{\alpha}\right)$ for $T^{\prime} M$ defines a unique section $M \rightarrow Y$ for which the pullbacks of $\left(\omega, \omega^{\alpha}\right)$ coincide with $\left(\theta, \theta^{\alpha}\right)$ and the pullback of $\varphi$ vanishes. As in [W78], we use this section to pull back the forms (3.11) to $M$. We shall use the same notation for the pulled back forms on $M$ (that now depend on the choice of the admissible coframe). With this convention, we have

$$
\theta=\omega, \quad \theta^{\alpha}=\omega^{\alpha}, \quad \varphi=0
$$

on $M$. Now, in view of [W78, (3.8)], the pulled back tangential pseudoconformal curvature tensor $S_{\alpha}{ }_{\mu \bar{\nu}}$ can be obtained from the tangential pseudo- 
hermitian curvature tensor $R_{\alpha}{ }^{\beta}{ }_{\mu \bar{\nu}}$ in (3.8) by

$S_{\alpha \bar{\beta} \mu \bar{\nu}}=R_{\alpha \bar{\beta} \mu \bar{\nu}}-\frac{R_{\alpha \bar{\beta}} g_{\mu \bar{\nu}}+R_{\mu \bar{\beta}} g_{\alpha \bar{\nu}}+R_{\alpha \bar{\nu}} g_{\mu \bar{\beta}}+R_{\mu \bar{\nu}} g_{\alpha \bar{\beta}}}{n+2}+\frac{R\left(g_{\alpha \bar{\beta}} g_{\mu \bar{\nu}}+g_{\alpha \bar{\nu}} g_{\mu \bar{\beta}}\right)}{(n+1)(n+2)}$

where

$$
R_{\alpha \bar{\beta}}:=R_{\mu}{ }^{\mu}{ }_{\alpha \bar{\beta}} \text { and } R:=R_{\mu}{ }^{\mu}
$$

are respectively the pseudohermitian Ricci and scalar curvature of $(M, \theta)$. Formula (3.16) expresses the fact that $S_{\alpha \bar{\beta} \mu \bar{\nu}}$ is the "traceless component" of $R_{\alpha \bar{\beta} \mu \bar{\nu}}$ with respect to the natural decomposition of the space of all tensors $T_{\alpha \bar{\beta} \mu \bar{\nu}}$ with the symmetry condition as for $S_{\alpha \bar{\beta}} \mu \bar{\nu}$ in (3.14) into the direct sum of the subspace of such tensors of trace zero (i.e. $T_{\mu}{ }^{\mu}{ }_{\alpha \bar{\beta}}=0$ ) and the subspace of "multiples of the Levi form", i.e. tensors of the form

$$
T_{\alpha \bar{\beta}} \mu \bar{\nu}=H_{\alpha \bar{\beta}} g_{\mu \bar{\nu}}+H_{\mu \bar{\beta}} g_{\alpha \bar{\nu}}+H_{\alpha \bar{\nu}} g_{\mu \bar{\beta}}+H_{\mu \bar{\nu}} g_{\alpha \bar{\beta}},
$$

where $\left(H_{\alpha \bar{\beta}}\right)$ is any Hermitian matrix. We shall call two tensors as above conformally equivalent if their difference is of the form (3.18). In this terminology, the right hand side of (3.16) (together with (3.17)) gives for any tensor $R_{\alpha \bar{\beta} \mu \bar{\nu}}$ (with the above symmetry relations) its traceless component which is the unique tensor of trace zero that is conformally equivalent to $R_{\alpha \bar{\beta} \mu \bar{\nu}}$.

The following result establishes relations between pseudoconformal and pseudohermitian connection forms and is alluded to in [W78].

Proposition 3.1. Let $M$ be a strictly pseudoconvex CR-manifold of hypersurface type of CR-dimension $n$, let $\omega_{\beta}{ }^{\alpha}$, $\tau^{\alpha}$ be defined by (3.6-3.7) with respect to an admissible coframe $\left(\theta, \theta^{\alpha}\right)$ and let $\varphi_{\beta}{ }^{\alpha}, \varphi^{\alpha}, \psi$ be the forms in (3.11) pulled back to $M$ using $\left(\theta, \theta^{\alpha}\right)$ as above. Then we have the following relations:

$\varphi_{\beta}{ }^{\alpha}=\omega_{\beta}{ }^{\alpha}+D_{\beta}{ }^{\alpha} \theta, \quad \varphi^{\alpha}=\tau^{\alpha}+D_{\mu}{ }^{\alpha} \theta^{\mu}+E^{\alpha} \theta, \quad \psi=i E_{\mu} \theta^{\mu}-i E_{\bar{\nu}} \theta^{\bar{\nu}}+B \theta$

where

$$
\begin{aligned}
& D_{\alpha \bar{\beta}}:=\frac{i R_{\alpha \bar{\beta}}}{n+2}-\frac{i R g_{\alpha \bar{\beta}}}{2(n+1)(n+2)}, \\
& E^{\alpha}:=\frac{2 i}{2 n+1}\left(A_{; \mu}^{\alpha \mu}-D_{; \bar{\nu}}^{\bar{\nu} \alpha},\right. \\
& B:=\frac{1}{n}\left({E^{\mu}}_{; \mu}+E_{; \bar{\nu}}^{\bar{\nu}}-2 A^{\beta \mu} A_{\beta \mu}+2 D^{\bar{\nu} \alpha} D_{\bar{\nu} \alpha}\right) .
\end{aligned}
$$


Proof. The formulas for $\varphi_{\beta}{ }^{\alpha}$ and $D_{\alpha \bar{\beta}}$ were proved in [W78]. The formula for $\varphi^{\alpha}$ follows from the third equation in (3.12) and (3.7). Indeed, these two equations yield

$$
\theta^{\alpha} \wedge \omega_{\alpha}^{\beta}+\theta \wedge \tau^{\beta}=\theta^{\alpha} \wedge \varphi_{\alpha}^{\beta}+\theta \wedge \varphi^{\beta}
$$

Substituting the formula for $\varphi_{\beta}{ }^{\alpha}$ in (3.19), we obtain

$$
\theta \wedge \tau^{\beta}=D_{\alpha}^{\beta} \theta^{\alpha} \wedge \theta+\theta \wedge \varphi^{\beta}
$$

which implies the formula for $\varphi^{\alpha}$ in (3.19) with some $E^{\alpha}$. Similarly, the formula for $\psi$ in (3.19) with some $B$ follows from equating the coefficients of $\theta$ in the pulled back fourth equation of (3.12) and using (3.15) (whence $d \varphi=0$ on $M)$.

To obtain the formula for $E^{\alpha}$ in (3.20), we substitute the formulas (3.19) for $\varphi_{\beta}{ }^{\alpha}, \varphi^{\alpha}, \psi$ in the pulled back sixth equation of (3.12) and use (3.3), (3.7), the covariant derivative (3.9) (and the analogue for $D_{\beta}{ }^{\alpha}$ ) and the formula for $\Phi^{\alpha}$ in (3.13):

$\nabla A^{\alpha} \bar{\nu}_{\nu} \theta^{\bar{\nu}}+\nabla D_{\beta}^{\alpha} \wedge \theta^{\beta}+i g_{\mu \bar{\nu}} E^{\alpha} \theta^{\mu} \wedge \theta^{\bar{\nu}}=-\frac{1}{2} \psi \wedge \theta^{\alpha}+V_{\mu \bar{\nu}}^{\alpha} \theta^{\mu} \wedge \theta^{\bar{\nu}} \bmod \theta$.

By identifying the coefficient in front of $\theta^{\mu} \wedge \theta^{\bar{\nu}}$ in (3.22) and using the formula for $\psi$ in (3.19), we obtain

$$
A_{\bar{\nu} ; \mu}^{\alpha}-D_{\mu}{ }^{\alpha}{ }_{\bar{\nu}}+i g_{\mu \bar{\nu}} E^{\alpha}=-\frac{1}{2} i E_{\bar{\nu}} \delta_{\mu}^{\alpha}+V_{\mu \bar{\nu}}^{\alpha} .
$$

The formula for $E^{\alpha}$ in (3.20) is now obtained by summing over $\mu$ and $\bar{\nu}$ and using the trace condition $V^{\alpha}{ }_{\mu}^{\mu}=0$. Similarly, the formula for $B$ follows by substituting the formula for $\psi$ in pulled back last equation of $(3.12)$ ( $\bmod$ $\theta$ ) and using the trace condition $P_{\nu}^{\nu}=0$.

\section{Submanifolds of CR-manifolds; the second fundamental form.}

Let $M$ be a strictly pseudoconvex CR-manifold of dimension $2 n+1$ as before and $f: M \rightarrow \hat{M}$ be an CR-immersion of $M$ into another strictly pseudoconvex CR-manifold $\hat{M}$ of dimension $2 \hat{n}+1$, with rank $\hat{n}$ CR-bundle $\hat{\mathcal{V}}$. Our arguments in the sequel will be of local nature and hence we shall assume $f$ to be an embedding. We shall use a ${ }^{\wedge}$ to denote objects associated to $\hat{M}$. Capital Latin indices $A, B$, etc, will run over the set $\{1,2, \ldots, \hat{n}\}$ 
whereas Greek indices $\alpha, \beta$, etc, will run over $\{1,2, \ldots, n\}$ as above. Moreover, we shall let small Latin indices $a, b$, etc, run over the complementary set $\{n+1, n+2, \ldots, \hat{n}\}$. Since $\hat{M}$ is strictly pseudoconvex and $f$ an embedding, it is well known that for any contact form $\hat{\theta}$ on $\hat{M}$ the pullback $f^{*}(\hat{\theta})$ (which for a CR-mapping $f$ is always a section of $T^{0} M$ ) is nonvanishing and, hence, a contact form on $M$ (In general, $f^{*}(\hat{\theta})$ may vanish, e.g. if $f(M)$ is contained in a complex-analytic subvariety of $\hat{M})$. We shall always choose the coframe $\left(\hat{\theta}, \hat{\theta}^{A}\right)$ on $\hat{M}$ such that the pullback of $\left(\hat{\theta}, \hat{\theta}^{\alpha}\right)$ is a coframe for $M$ and hence drop the ${ }^{\wedge}$ over the frames and coframes if there is no ambiguity. It will be clear from the context if a form is pulled back to $M$ or not.

We shall identify $M$ with the submanifold $f(M)$ of $\hat{M}$ and write $M \subset \hat{M}$. Then $\mathcal{V}$ becomes a rank $n$ subbundle of $\hat{\mathcal{V}}$ along $M$. It follows that the (real) codimension of $M$ in $\hat{M}$ is $2(\hat{n}-n)$ and that there is a rank $(\hat{n}-n)$ subbundle $N^{\prime} M$ of $T^{\prime} \hat{M}$ along $M$ consisting of 1 -forms on $\hat{M}$ whose pullbacks to $M$ (under $f$ ) vanish. We shall call $N^{\prime} M$ the holomorphic conormal bundle of $M$ in $\hat{M}$. We shall say that the pseudohermitian structure $(\hat{M}, \hat{\theta})$ (or simply $\hat{\theta})$ is admissible for the pair $(M, \hat{M})$ if the characteristic vector field $\hat{T}$ of $\hat{\theta}$ is tangent to $M$ (and hence coincides with the characteristic vector field of the pullback of $\hat{\theta}$ ). This is equivalent, as the reader can easily verify, to requiring that for any admissible coframe $\left(\hat{\theta}, \hat{\theta}^{A}\right)$ on $\hat{M}$, where $A=1, \ldots, \hat{n}$, the holomorphic conormal bundle $N^{\prime} M$ is spanned by suitable linear combinations of the $\hat{\theta}^{A}$. It is easily seen that not all contact forms $\hat{\theta}$ are admissible for $(M, \hat{M})$. However, we have the following statement:

Lemma 4.1. Let $M \subset \hat{M}$ be as above. Then any contact form on $M$ can be extended to a contact form $\theta$ in a neighborhood of $M$ in $\hat{M}$ such that $\theta$ is admissible for $(M, \hat{M})$. Moreover, the 1-jet of $\theta$ is uniquely determined on $M$.

Proof. Let $\theta$ be any fixed extension of the given contact form on $M$ to a neighborhood of $M$ in $\hat{M}$. Any other extension is clearly of the form $\tilde{\theta}=u \theta$, where $u$ is a smooth function on $\hat{M}$ near $M$ with $\left.u\right|_{M} \equiv 1$. Let $T$ be the characteristic vector field of the restriction of $\theta$ to $M$. Then $\tilde{\theta}$ is admissible for $(M, \hat{M})$ if and only if $T\lrcorner d \tilde{\theta}=0$, i.e. if $T\lrcorner d \theta-d u=0$ along $M$. By the assumptions, the latter identity holds when pulled back to $M$. Now it is clear that there exists unique choice of $d u$ along $M$ for which it holds also in the normal direction. The required function $u$ can be now constructed in local coordinate charts and glued together via partition of unity. The proof is complete. 
By taking admissible coframes as in $\S 3$ and using the Gram-Schmidt algorithm, we obtain the following corollary, where we take a little more care to distinguish between $M$ and its image $f(M)$ in $\hat{M}$.

Corollary 4.2. Let $M$ and $\hat{M}$ be strictly pseudoconvex CR-manifolds of dimensions $2 n+1$ and $2 \hat{n}+1$ respectively and $f: M \rightarrow \hat{M}$ be a $C R$-embedding. If $\left(\theta, \theta^{\alpha}\right)$ is any admissible coframe on $M$, then in a neighborhood of any point $\hat{p} \in f(M)$ in $\hat{M}$ there exists an admissible coframe $\left(\hat{\theta}, \hat{\theta}^{A}\right)$ on $\hat{M}$ with $f^{*}\left(\hat{\theta}, \hat{\theta}^{\alpha}, \hat{\theta}^{a}\right)=\left(\theta, \theta^{\alpha}, 0\right)$. In particular, $\hat{\theta}$ is admissible for the pair $(f(M), \hat{M})$, i.e. the characteristic vector field $\hat{T}$ is tangent to $f(M)$. If the Levi form of $M$ with respect to $\left(\theta, \theta^{\alpha}\right)$ is $\delta_{\alpha \bar{\beta}}$, then $\left(\hat{\theta}, \hat{\theta}^{A}\right)$ can be chosen such that the Levi form of $\hat{M}$ relative to it is also $\delta_{A \bar{B}}$. With this additional property, the coframe $\left(\hat{\theta}, \hat{\theta}^{A}\right)$ is uniquely determined along $M$ up to unitary transformations in $\mathrm{U}(n) \times \mathrm{U}(\hat{n}-n)$.

Let us fix an admissible coframe $\left(\theta, \theta^{\alpha}\right)$ on $M$ and let $\left(\hat{\theta}, \hat{\theta}^{A}\right)$ be an admissible coframe on $\hat{M}$ near $f(M)$. We shall say that $\left(\hat{\theta}, \hat{\theta}^{A}\right)$ is adapted to $\left(\theta, \theta^{\alpha}\right)$ on $M$ (or simply to $M$ if the coframe on $M$ is understood) if it satisfies the conclusion of Corollary 4.2 with the requirement there for the Levi form.

The fact that $\left(\theta, \theta^{A}\right)$ (where we omit $\mathrm{a}^{\wedge}$ ) is adapted to $M$ implies, in view of the construction (3.6), that if the pseudohermitian connection matrix of $(\hat{M}, \theta)$ is $\hat{\omega}_{B}{ }^{A}$, then that of $(M, \theta)$ is (the pullback of) $\hat{\omega}_{\beta}{ }^{\alpha}$. Similarly, the pulled back torsion matrix $\hat{\tau}^{\alpha}$ is $\tau^{\alpha}$. Hence omitting a over these pullbacks will not cause any ambiguity and we shall do it in the sequel. By our normalization of the Levi form, the second equation in (3.6) reduces to

$$
\omega_{B \bar{A}}+\omega_{\bar{A} B}=0
$$

where as before $\omega_{\bar{A} B}=\overline{\omega_{A \bar{B}}}$.

The matrix of 1 -forms $\left(\omega_{\alpha}^{b}\right)$ pulled back to $M$ defines the second fundamental form of $M$ (or more precisely of the embedding $f$ ). It was used e.g. in [W79, Fa90] in the study of mapping problems. Since $\theta^{b}$ is 0 on $M$, we deduce by using equation (3.7) that, on $M$,

$$
\omega_{\alpha}^{b} \wedge \theta^{\alpha}+\tau^{b} \wedge \theta=0
$$

which implies that

$$
\omega_{\alpha}^{b}=\omega_{\alpha \beta}^{b} \theta^{\beta}, \quad \omega_{\alpha \beta}^{b}=\omega_{\beta}^{b}{ }_{\alpha}, \quad \tau^{b}=0
$$


We now relate so defined intrinsic second fundamental form $\left(\omega_{\alpha}^{b}{ }^{b}\right)$ with the extrinsic one defined in $\S 2$ in case $\hat{M}$ is embedded as a real hypersurface in $\mathbb{C}^{\hat{n}+1}$. Given any admissible contact form $\theta$ for $(M, \hat{M})$, we can choose a defining function of $\hat{M}$ near a point $p=\hat{p} \in M$ such that $\theta=i \bar{\partial} \hat{\rho}$ on $\hat{M}$, i.e. in local coordinates $Z^{\prime}$ in $\mathbb{C}^{\hat{n}+1}$ vanishing at $p$ we have

$$
\theta=i \sum_{k=1}^{\hat{n}+1} \frac{\partial \hat{\rho}}{\partial \bar{Z}_{k}^{\prime}} d \bar{Z}_{k}^{\prime}
$$

where we pull back the forms $d \bar{Z}_{1}^{\prime}, \ldots, d \bar{Z}_{\hat{n}+1}^{\prime}$ to $\hat{M}$. Given further a coframe $\left(\theta, \theta^{A}\right)$ on $\hat{M}$ near $p$ adapted to $M$ and its dual frame $\left(T, L_{A}\right)$, we have

$$
\left.L_{\beta}\left(\hat{\rho}_{\bar{Z}^{\prime}} \circ f\right)=-i L_{\beta}\right\lrcorner d \theta=g_{\beta \bar{C}} \theta^{\bar{C}}=g_{\beta \bar{\gamma}} \theta^{\bar{\gamma}} .
$$

Recall that we are in fact assuming that the Levi form has been normalized, i.e. $g_{A \bar{B}}=\delta_{A \bar{B}}$, even though we retain the notation $g_{A \bar{B}}$. Conjugating (4.5) we see that the subspace $E_{1}(p) \subset T_{p}^{\prime} \mathbb{C}^{\hat{n}+1}$ in $(2.1)$ is spanned by $\left(\theta, \theta^{\alpha}\right)$, where we use the standard identification $T_{p}^{\prime} \hat{M} \cong T_{p}^{\prime} \mathbb{C}^{\hat{n}+1}$. Applying $L_{\alpha}$ to both sides of (4.5) and using the analogue of (3.6) for $\hat{M}$ and (4.3), we conclude that

$$
\left.L_{\alpha} L_{\beta}\left(\hat{\rho}_{\bar{Z}^{\prime}} \circ f\right)=g_{\beta \bar{\gamma}} L_{\alpha}\right\lrcorner d \theta^{\bar{\gamma}}=-\omega_{\bar{a} \beta \alpha} \theta^{\bar{a}}=\omega_{\alpha \bar{a} \beta} \theta^{\bar{a}} \quad \bmod \theta, \theta^{\bar{\alpha}},
$$

where we have used (4.1) for the last identity. Comparing with the extrinsic definition of the second fundamental form (2.3) and identifying the spaces in (2.3) and (2.4) via the Levi form of $\hat{M}$ as explained in $\S 2$, we conclude that

$$
\Pi\left(L_{\alpha}, L_{\beta}\right)=\omega_{\alpha}^{a}{ }_{\beta} L_{a},
$$

where with have identified $L_{a}$ with its equivalence classe in $T_{\hat{p}}^{1,0} \hat{M} / T_{p}^{1,0} M$. Conjugating (4.6) and comparing with (2.1) we see that the space $E_{2}=E_{2}(p)$ is spanned (via the identification above) by the forms

$$
\theta, \theta^{\alpha}, \omega_{\bar{\alpha} a \bar{\beta}} \theta^{a} .
$$

To interpret the higher order spaces $E_{k}(p)$ in terms of the second fundamental form, we shall need the covariant differentials of the second fundamental form with respect to the induced pseudoconformal connection, which will be introduced in section 6 . The discussion will therefore be postponed until section 9 . 
As a byproduct of the relation (4.7) we see that the bilinear map $T_{p}^{1,0} M \times$ $T_{p}^{1,0} M \rightarrow T_{p}^{1,0} \hat{M} / T_{p}^{1,0} M$ defined by $\left(\omega_{\alpha}{ }^{a} \beta\right)$ is independent of the choice of the adapted coframe $\left(\theta, \theta^{A}\right)$ in case $\hat{M}$ is locally CR-embeddable (in $\left.\mathbb{C}^{\hat{n}+1}\right)$. More generally, if $\hat{M}$ is not CR-embeddable, one can still use approximate embeddings constructed in [KZ01] (or direct computation of the change of $\left.\omega_{\alpha}^{a} \beta\right)$ to obtain the same conclusion.

\section{CR analogue of the Gauss equation and applications.}

The classical Gauss equation in Riemannian geometry relates the Riemannian curvature tensors of a manifold and its submanifold with the second fundamental form composed with the Riemannian metric (see e.g. [KN69]). Our goal in this section will be to establish the pseudohermitian and pseudoconformal analogues of the Gauss equation and to apply them to obtain rigidity properties of the second fundamental form.

As before we fix a coframe $\left(\theta, \theta^{A}\right)$ adapted to $M$. We first compare pseudohermitian curvature tensors $R_{\alpha}{ }^{\beta}{ }_{\mu \bar{\nu}}$ and $\hat{R}_{A}{ }^{B} C \bar{D}$ of $(\hat{M}, \theta)$ and $(M, \theta)$ respectively. By comparing (3.8) and the corresponding equation for $\hat{R}_{A}{ }_{C} \bar{D}$ pulled back to $M$ and using $\hat{\omega}_{\alpha}{ }^{\beta}=\omega_{\alpha}{ }^{\beta}, \hat{\tau}^{\alpha}=\tau^{\alpha}$ and $\hat{W}_{\alpha}{ }^{\beta}{ }_{\mu}=W_{\alpha}{ }^{\beta}{ }_{\mu}$ as a consequence of (3.10), we conclude that on $M$,

$$
\hat{R}_{\alpha}{ }^{\beta}{ }_{\mu \bar{\nu}} \theta^{\mu} \wedge \theta^{\bar{\nu}}+\omega_{\alpha}{ }^{a} \wedge \omega_{a}{ }^{\beta}=R_{\alpha}{ }^{\beta}{ }_{\mu \bar{\nu}} \theta^{\mu} \wedge \theta^{\bar{\nu}} .
$$

By using the symmetry (4.1), we conclude that, on $M$, we have

$$
\hat{R}_{\alpha \bar{\beta} \mu \bar{\nu}} \theta^{\mu} \wedge \theta^{\bar{\nu}}-\omega_{\alpha}^{a} \wedge \omega_{\bar{\beta} a}=R_{\alpha \bar{\beta} \mu \bar{\nu}} \theta^{\mu} \wedge \theta^{\bar{\nu}} .
$$

This can also be written, in view of (4.3), after equating the coefficients of $\theta^{\mu} \wedge \theta^{\bar{\nu}}$ as

$$
\hat{R}_{\alpha \bar{\beta} \mu \bar{\nu}}=R_{\alpha \bar{\beta} \mu \bar{\nu}}+g_{a \bar{b}} \omega_{\alpha}{ }^{a}{ }_{\mu} \omega_{\bar{\beta}}{ }^{\bar{b}} \bar{\nu} .
$$

The identity (5.3) relates the tangential pseudohermitian curvature tensors of $M$ and $\hat{M}$ with the second fundamental form of the embedding $M$ into $\hat{M}$ and hence can be viewed as pseudohermitian analogue of the Gauss equation. We state it in an invariant form using the previously established relation (4.7) between the extrinsic and intrinsic second fundamental forms $\Pi$ and $\left(\omega_{\alpha}{ }^{a} \beta\right)$ given respectively by $(2.3-2.4)$ and (4.3). For this, we view both pseudohermitian curvature tensors as $\mathbb{R}$-multilinear functions

$$
R, \hat{R}: T^{1,0} M \times T^{1,0} M \times T^{1,0} M \times T^{1,0} M \rightarrow \mathbb{C}
$$

that are $\mathbb{C}$-linear in the even and $\mathbb{C}$-antilinear in the odd numbered arguments. We further identify the quotient space $T_{p}^{1,0} \hat{M} / T_{p}^{1,0} M$ for $p \in M$ with 
the orthogonal complement of $T_{p}^{1,0} M$ in $T_{p}^{1,0} \hat{M}$ with respect to the Levi form of $\hat{M}$ relative to $\theta$ and then use this Levi form to define a canonical Hermitian scalar product $\langle$,$\rangle on T_{p}^{1,0} \hat{M} / T_{p}^{1,0} M$. The identity (5.3) yields now the following statement.

Proposition 5.1 (Pseudohermitian Gauss equation). Let $M \subset \hat{M}$ be as above and $\theta$ be a contact form on $\hat{M}$ that is admissible for the pair $(M, \hat{M})$. Then, for every $p \in M$, the following holds:

$$
\hat{R}(X, Y, Z, V)=R(X, Y, Z, V)+\langle\Pi(X, Z), \Pi(Y, V)\rangle, \quad X, Y, Z, V \in T_{p}^{1,0} M
$$

We next turn to a pseudoconformal analogue of the Gauss equation. It follows immediately from (5.3) and (5.4) by taking traceless components of both sides as discussed in $\S 3$. We write $\left[T_{\alpha \bar{\beta} \mu \bar{\nu}}\right]$ for the traceless component of a tensor $T_{\alpha \bar{\beta}} \mu \bar{\nu}$ that can be computed by the formulas (3.16-3.17) (with $R_{\alpha \bar{\beta} \mu \bar{\nu}}$ replaced by $\left.T_{\alpha \bar{\beta} \mu \bar{\nu}}\right)$. Then $(3.16)$ can be rewritten as

$$
S_{\alpha \bar{\beta} \mu \bar{\nu}}=\left[R_{\alpha \bar{\beta} \mu \bar{\nu}}\right]
$$

By taking traceless components of both sides in (5.3) and using (5.5) we now obtain

$$
\left[\hat{R}_{\alpha \bar{\beta} \mu \bar{\nu}}\right]=S_{\alpha \bar{\beta} \mu \bar{\nu}}+\left[g_{a \bar{b}} \omega_{\alpha}{ }^{a}{ }_{\mu} \omega_{\bar{\beta}}{ }^{\bar{b}} \bar{\nu}\right] .
$$

Note that, in contrast to (5.5), the left hand side of (5.6) may not be, in general, equal to $\hat{S}_{\alpha \bar{\beta} \mu \bar{\nu}}$ which is the (restriction of the) traceless component of $\hat{R}_{A \bar{B} C \bar{D}}$ with respect to the indices running from 1 to $\hat{n}$. However, we claim that $\left[\hat{R}_{\alpha \bar{\beta} \mu \bar{\nu}}\right]=\left[\hat{S}_{\alpha \bar{\beta} \mu \bar{\nu}}\right]$. Indeed, according to the decomposition into zero trace component and a multiple of the Levi form $\left(g_{A \bar{B}}\right)$ on $\hat{M}$, the tensors $\hat{R}_{A \bar{B} C \bar{D}}$ and $\hat{S}_{A \bar{B} C \bar{D}}$ are conformally equivalent with respect to the Levi form $\left(g_{A \bar{B}}\right)$, i.e. their difference is of the form analogous to (3.18) with Greek indices replaced by capital Latin ones. Since the Levi form of $\hat{M}$ restricts to that of $M$, the restrictions $\hat{R}_{\alpha \bar{\beta}} \mu \bar{\nu}$ and $\hat{S}_{\alpha \bar{\beta} \mu \bar{\nu}}$ are conformally equivalent with respect to $\left(g_{\alpha \bar{\beta}}\right)$ and the claim follows. Hence (5.6) immediately yields the desired relation between the tangential preudoconformal curvature tensors of $M$ and $\hat{M}$ and the second fundamental form:

$$
\left[\hat{S}_{\alpha \bar{\beta} \mu \bar{\nu}}\right]=S_{\alpha \bar{\beta} \mu \bar{\nu}}+\left[g_{a \bar{b}} \omega_{\alpha}^{a}{ }_{\mu} \omega_{\bar{\beta}}^{\bar{b}} \bar{\nu}\right]
$$


or, using formulas (3.16-3.17) for the traceless part,

$$
\begin{aligned}
S_{\alpha \bar{\beta} \mu \bar{\nu}}= & \hat{S}_{\alpha \bar{\beta} \mu \bar{\nu}}-\frac{\hat{S}_{\gamma}{ }_{\alpha \bar{\beta}} g_{\mu \bar{\nu}}+\hat{S}_{\gamma}{ }_{\mu \bar{\beta}} g_{\alpha \bar{\nu}}+\hat{S}_{\gamma}{ }^{\gamma}{ }_{\alpha \bar{\nu}} g_{\mu \bar{\beta}}+\hat{S}_{\gamma}{ }^{\gamma}{ }_{\mu \bar{\nu}} g_{\alpha \bar{\beta}}}{n+2} \\
& +\frac{\hat{S}_{\gamma}{ }^{\gamma} \delta^{\delta}\left(g_{\alpha \bar{\beta}} g_{\mu \bar{\nu}}+g_{\alpha \bar{\nu}} g_{\mu \bar{\beta}}\right)}{(n+1)(n+2)}-g_{a \bar{b}} \omega_{\alpha}{ }^{a}{ }_{\mu} \omega_{\bar{\beta}} \bar{b}_{\bar{\nu}} \\
& +\frac{\omega_{\gamma}{ }^{a}{ }_{\alpha} \omega^{\gamma}{ }_{a \bar{\beta}} g_{\mu \bar{\nu}}+\omega_{\gamma}{ }^{a}{ }_{\mu} \omega^{\gamma}{ }_{a \bar{\beta}} g_{\alpha \bar{\nu}}+\omega_{\gamma}{ }^{a}{ }_{\alpha} \omega^{\gamma}{ }_{a \bar{\nu}} g_{\mu \bar{\beta}}+\omega_{\gamma}{ }^{a}{ }_{\mu} \omega^{\gamma}{ }_{a \bar{\nu}} g_{\alpha \bar{\beta}}}{n+2} \\
& -\frac{\omega_{\gamma}{ }^{a}{ }_{\delta} \omega^{\gamma}{ }_{a}{ }^{\delta}\left(g_{\alpha \bar{\beta}} g_{\mu \bar{\nu}}+g_{\alpha \bar{\nu}} g_{\mu \bar{\beta}}\right)}{(n+1)(n+2)} .
\end{aligned}
$$

When $\hat{n}=n+1$ and $\hat{M}$ is a sphere (so that $\hat{S}_{\alpha \bar{\beta} \mu \bar{\nu}} \equiv 0$ ), the identity (5.8) reduces to that obtained by Webster [W79, (2.14)]. As for the pseudohermitian curvature, we also view the pseudoconformal curvature tensors (as well as their zero trace components) as $\mathbb{R}$-multilinear functions

$$
S, \hat{S}: T^{1,0} M \times T^{1,0} M \times T^{1,0} M \times T^{1,0} M \rightarrow \mathbb{C}
$$

but now they are independent of $\theta$. Then, with the above notation, (5.7) yields the following statement.

Proposition 5.2 (Pseudoconformal Gauss equation). For $M \subset \hat{M}$ as above and every $p \in M$, the following holds:

$$
[\hat{S}(X, Y, Z, V)]=S(X, Y, Z, V)+[\langle\Pi(X, Z), \Pi(Y, V)\rangle], \quad X, Y, Z, V \in T_{p}^{1,0} M .
$$

In the rest of this section we apply the Gauss equations (5.7-5.9) to the case when $\hat{M}$ is the sphere and hence $\hat{S}_{A \bar{B} C \bar{D}} \equiv 0$. Based on a lemma due to the second author [Hu99], we show that the second fundamental form in this case is uniquely determined up to unitary transformations by the tangential pseudoconformal curvature $S_{\alpha \bar{\beta}} \mu \bar{\nu}$ under suitable restrictions on the dimensions $n$ and $\hat{n}$. We begin with an algebraic lemma.

Lemma 5.3. Let $\Pi: \mathbb{C}^{n} \times \mathbb{C}^{n} \rightarrow \mathbb{C}^{\hat{n}-n}$ be a $\mathbb{C}$-bilinear map and $\langle\cdot, \cdot\rangle$ an Hermitian scalar product on $\mathbb{C}^{\hat{n}-n}$. Denote by $S:=[\langle\Pi(\cdot, \cdot), \Pi(\cdot, \cdot)\rangle]$ the traceless component with respect to a fixed Hermitian scalar product $(\cdot, \cdot)$ on $\mathbb{C}^{n}$ as above.

(i) If $\Pi \equiv 0$, then also $S \equiv 0$. The converse also holds under the assumption $\hat{n}-n<n$. 
(ii) If $\hat{n}-n<n / 2$, then $\Pi$ is uniquely determined by $S$ up to unitary transformations of $\mathbb{C}^{\hat{n}-n}$, i.e. if $\tilde{\Pi}$ is another form, then $S=[\langle\tilde{\Pi}(\cdot, \cdot), \tilde{\Pi}(\cdot, \cdot)\rangle]$ if and only if $\tilde{\Pi}=U \circ \Pi$ for some $U \in \mathrm{GL}(\hat{n}-n)$ preserving $\langle\cdot, \cdot\rangle$.

Proof. The first statement of (i) is trivial. By definition of the traceless component, $S \equiv 0$ implies $\langle\Pi(X, X), \Pi(X, X)\rangle=(X, X) H(X, X)$ for some Hermitian form $H(\cdot, \cdot)$ and all $X \in \mathbb{C}^{n}$. Then the second statement of (i) follows directly from [Hu99, Lemma 3.2]. To show (ii) observe that, for $\Pi$ and $\tilde{\Pi}$ as in the lemma, one similarly has

$$
\langle\Pi(X, X), \Pi(X, X)\rangle-\langle\tilde{\Pi}(X, X), \tilde{\Pi}(X, X)\rangle=(X, X) H(X, X) .
$$

The restriction $\hat{n}-n<n / 2$ permits now to conclude from [Hu99, Lemma 3.2] that $H(X, X) \equiv 0$. By polarizing the left hand side and using the symmetries, we obtain

$$
\langle\Pi(X, Y), \Pi(Z, V)\rangle=\langle\tilde{\Pi}(X, Y), \tilde{\Pi}(Z, V)\rangle, \quad X, Y, Z, V \in \mathbb{C}^{n},
$$

expressing the fact that the collections of vectors $(\Pi(X, Y))_{X, Y}$ and $(\tilde{\Pi}(X, Y))_{X, Y}$ have the same scalar products. Hence they can be transformed into each other by a transformation $U \in \operatorname{GL}(\hat{n}-n)$ preserving $\langle\cdot, \cdot\rangle$. Any such $U$ satisfies the required conclusion proving (ii).

Example 1.1 shows that (i) may not hold in case of equality $\hat{n}-n=n$. Furthermore, the conclusion of (ii) may not hold under the weaker inequality $\hat{n}-n<n$ that was enough to obtain (i):

Example 5.4. For $n:=2, \hat{n}:=3$, consider the standard scalar products $(\cdot, \cdot)$ on $\mathbb{C}^{2}$ and $\langle\cdot, \cdot\rangle$ on $\mathbb{C}$ and define $\Pi$ and $\tilde{\Pi}$ by their associated quadratic forms $Q:=4 z_{1}^{2}+z_{1} z_{2}+4 z_{2}^{2}$ and $\tilde{Q}:=4 z_{1}^{2}-z_{1} z_{2}+4 z_{2}^{2}$ respectively. Then the identity

$$
\left|4 z_{1}^{2}+z_{1} z_{2}+4 z_{2}^{2}\right|^{2}-\left|4 z_{1}^{2}-z_{1} z_{2}+4 z_{2}^{2}\right|^{2}=8\left(\left|z_{1}\right|^{2}+\left|z_{2}\right|^{2}\right)\left(z_{1} \bar{z}_{2}+\bar{z}_{1} z_{2}\right)
$$

implies that $[\langle\Pi(\cdot, \cdot), \Pi(\cdot, \cdot)\rangle]=[\langle\tilde{\Pi}(\cdot, \cdot), \tilde{\Pi}(\cdot, \cdot)\rangle]$. However, it is clear that the conclusion of (ii) does not hold for $\Pi$ and $\tilde{\Pi}$.

The transformation $U$ in Lemma 5.3 is clearly uniquely determined on the image of $\Pi$. We apply Lemma 5.3 to compare second fundamental forms $\Pi=\Pi_{p}$ and $\tilde{\Pi}=\tilde{\Pi}_{p}$ at a point $p \in M$ for two given embedding $f, \tilde{f}: M \rightarrow$ 
$\hat{M}$. Then, if the image of $\Pi$ is of constant dimension, in which case we say that $\Pi$ is of constant rank, the transformation $U$ can be chosen smoothly depending on $p$ (near any given point). We then obtain, as a consequence of (5.9-5.7) and Lemma 5.3, the following result.

Corollary 5.5. Let $f: M \rightarrow \hat{M}$ be a CR-embedding of a strictly pseudoconvex CR-manifold of dimension $2 n+1$ into the unit sphere $\hat{M}=\mathbb{S}$ in $\mathbb{C}^{\hat{n}+1}$. Denote by $\left(\omega_{\alpha}{ }^{a}\right)$ the second fundamental form matrix of $f$ relative to an admissible coframe $\left(\theta, \theta^{A}\right)$ on $\hat{M}$ adapted to $f(M)$.

(i) If $\omega_{\alpha}^{a} \beta \equiv 0$, then $M$ is locally CR-equivalent to the unit sphere in $\mathbb{C}^{n+1}$. The converse also holds under the assumption $\hat{n}-n<n$.

(ii) If $\hat{n}-n<n / 2$, then, for any $p \in M,\left(\omega_{\alpha}{ }^{a} \beta\right)(p)$ is uniquely determined by $\left(\theta, \theta^{\alpha}\right)$ up to unitary transformations of $\mathbb{C}^{\hat{n}-n}$, i.e. for any other CR-embedding $\tilde{f}: M \rightarrow \hat{M}$ and any coframe $\left(\tilde{\theta}, \tilde{\theta}^{A}\right)$ on $\hat{M}$ adapted to $\tilde{f}(M)$ with $\tilde{f}^{*}\left(\tilde{\theta}, \tilde{\theta}^{\alpha}\right)=f^{*}\left(\theta, \theta^{\alpha}\right)$, one has $\tilde{\omega}_{\alpha}{ }^{a}{ }_{\beta}(p)=\omega_{\alpha}{ }^{a}{ }_{\beta}(p)$ after possibly a unitary change of $\left(\tilde{\theta}^{a}\right)$ near $p$. Moreover, if $\left(\omega_{\alpha}^{a}{ }_{\beta}\right)$ is of constant rank near $p$, there there is such a unitary change of $\left(\tilde{\theta}^{a}\right)$ near $p$ for which $\tilde{\omega}_{\alpha}{ }^{a}=\omega_{\alpha}{ }^{a}$ near $p$.

\section{The induced pseudoconformal connection.}

Let now $\left(\theta, \theta^{A}\right)$ be an adapted coframe for the pair $(M, \hat{M})$ as above and let (3.11) be the 1-forms defining the parallelism on the bundle $Y$ over $M$ pulled back to $M$ by the coframe $\left(\theta, \theta^{\alpha}\right)$ as described in $\S 3$. We use ^ for the corresponding forms on $\hat{M}$ pulled back further to $M$, where the indices run from 1 to $\hat{n}$. Recall that $\left(\omega, \omega^{\alpha}, \omega^{\bar{\alpha}}\right)=\left(\hat{\omega}, \hat{\omega}^{\alpha}, \hat{\omega}^{\bar{\alpha}}\right)=\left(\theta, \theta^{\alpha}, \theta^{\bar{\alpha}}\right)$ and $\hat{\omega}^{a}=0$ on $M$. In view of Proposition 3.1, we do not expect $\left(\varphi_{\beta}{ }^{\alpha}, \varphi^{\alpha}, \psi\right)$ and $\left(\hat{\varphi}_{\beta}{ }^{\alpha}, \hat{\varphi}^{\alpha}, \hat{\psi}\right)$ to be equal. However, since $\hat{\omega}_{\beta}{ }^{\alpha}=\omega_{\beta}{ }^{\alpha}$ and $\tau^{\alpha}=\hat{\tau}^{\alpha}$ (see $\S 4$ ), Proposition 3.1 implies

$\hat{\varphi}_{\beta}{ }^{\alpha}=\varphi_{\beta}{ }^{\alpha}+C_{\beta}{ }^{\alpha} \theta, \quad \hat{\varphi}^{\alpha}=\varphi^{\alpha}+C_{\mu}{ }^{\alpha} \theta^{\mu}+F^{\alpha} \theta, \quad \hat{\psi}=\psi+i F_{\mu} \theta^{\mu}-i F_{\bar{\nu}} \theta^{\bar{\nu}}+A \theta$,

where

$$
C_{\beta}^{\alpha}:=\hat{D}_{\beta}^{\alpha}-D_{\beta}^{\alpha}, \quad F^{\alpha}:=\hat{E}^{\alpha}-E^{\alpha}, \quad A:=\hat{B}-B
$$

and $\hat{D}_{\beta}{ }^{\alpha}, \hat{E}^{\alpha}, \hat{B}$ are the analogues for $\hat{M}$ of the functions (3.20) restricted to $M$. 
Let us first compute $C_{\beta}{ }^{\alpha}$ using the pseudohermitian Gauss equation obtained in $\S 5$. In view of $(3.20)$, we have

$$
C_{\alpha \bar{\beta}}=\frac{i \hat{R}_{\alpha \bar{\beta}}}{\hat{n}+2}-\frac{i \hat{R} g_{\alpha \bar{\beta}}}{2(\hat{n}+1)(\hat{n}+2)}-\frac{i R_{\alpha \bar{\beta}}}{n+2}+\frac{i R g_{\alpha \bar{\beta}}}{2(n+1)(n+2)},
$$

where the pseudohermitian Ricci curvature tensors $\hat{R}_{\alpha \bar{\beta}}$ and $R_{\alpha \bar{\beta}}$ are related by

$$
R_{\alpha \bar{\beta}}=\hat{R}_{\mu}^{\mu}{ }_{\alpha \bar{\beta}}-\omega_{\mu}^{a}{ }_{\alpha} \omega^{\mu}{ }_{a \bar{\beta}}=\hat{R}_{\alpha \bar{\beta}}-\hat{R}_{a}^{a}{ }_{\alpha \bar{\beta}}-\omega_{\mu}^{a}{ }_{\alpha} \omega^{\mu}{ }_{a \bar{\beta}},
$$

where we have used the contraction of (5.3). The pseudohermitian scalar curvature of $R$ is now obtained by further contracting (6.3):

$$
R=\hat{R}_{\mu}{ }^{\mu}-\hat{R}_{a}^{a}{ }^{\mu}{ }^{\mu}-\omega_{\mu}{ }^{a}{ }_{\nu} \omega^{\mu}{ }_{a}^{\nu} .
$$

Substituting (6.3-6.4) into (6.2), we obtain

$$
\begin{aligned}
C_{\alpha \bar{\beta}}= & \frac{i \omega_{\mu}{ }^{a}{ }_{\alpha} \omega^{\mu}{ }_{a \bar{\beta}}}{n+2}+\frac{i \hat{R}_{a}{ }^{a} \alpha \bar{\beta}}{n+2}+\left(\frac{1}{\hat{n}+2}-\frac{1}{n+2}\right) i \hat{R}_{\alpha \bar{\beta}} \\
& -\frac{i \omega_{\mu}{ }^{a}{ }_{\nu} \omega^{\mu}{ }_{a}{ }^{\nu} g_{\alpha \bar{\beta}}}{2(n+1)(n+2)}-\frac{i\left(\hat{R}_{a}{ }^{a}{ }_{\mu}{ }^{\mu}+\hat{R}_{\mu}{ }^{\mu}\right) g_{\alpha \bar{\beta}}}{2(n+1)(n+2)}-\frac{i \hat{R} g_{\alpha \bar{\beta}}}{2(\hat{n}+1)(\hat{n}+2)} .
\end{aligned}
$$

We next express the contractions of the (tangential) pseudohermitian tensor of $\hat{M}$ appearing here in terms of its (tangential) pseudoconformal curvature tensor and the (pseudohermitian) Ricci and scalar curvature. For this, we contract the analogue of (3.16) (using the analogue of (3.17)) for $\hat{M}$ over $a=n+1, \ldots, \hat{n}$, and restrict as before to the situation where $g_{A \bar{B}}=\delta_{A \bar{B}}$ (so that, in particular, $g_{a \bar{\mu}}=0$ ):

$$
\hat{R}_{a}{ }^{a}{ }_{\alpha \bar{\beta}}=\hat{S}_{a}{ }^{a}{ }_{\alpha \bar{\beta}}+\frac{\hat{R}_{a}{ }^{a} g_{\alpha \bar{\beta}}+(\hat{n}-n) \hat{R}_{\alpha \bar{\beta}}}{\hat{n}+2}-\frac{(\hat{n}-n) \hat{R} g_{\alpha \bar{\beta}}}{(\hat{n}+1)(\hat{n}+2)} .
$$

Contracting again for $\mu=1, \ldots, n$, we further obtain

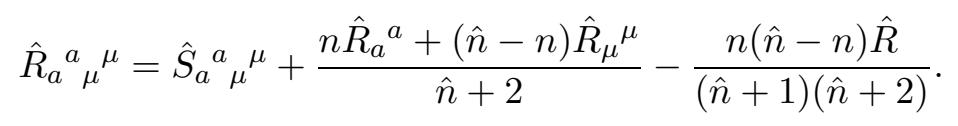

After substituting (6.6-6.7) in (6.5) and simplifying, we obtain

$$
C_{\alpha \bar{\beta}}=\frac{i\left(\hat{S}_{a}^{a}{ }_{\alpha \bar{\beta}}+\omega_{\mu}{ }^{a}{ }_{\alpha} \omega^{\mu}{ }_{a \bar{\beta}}\right)}{n+2}-\frac{i\left(\hat{S}_{a}{ }^{a} \mu^{\mu}+\omega_{\mu}{ }^{a}{ }_{\nu} \omega^{\mu}{ }_{a}{ }^{\nu}\right) g_{\alpha \bar{\beta}}}{2(n+1)(n+2)} .
$$


Thus the coefficients $C_{\alpha \bar{\beta}}$ are completely determined by the second fundamental form $\omega_{\alpha}{ }^{a} \beta$ and the tangential pseudoconformal curvature tensor $\hat{S}_{A \bar{B} C \bar{D}}$ of $\hat{M}$ pulled back to $M$.

Let us proceed to determine the $F^{\alpha}$. We compute $d \hat{\varphi}^{\alpha}$ modulo $\theta$ by differentiating the second equation in (6.1) and using the sixth structure equation in (3.12) pulled back to $M$ and (3.15):

$d \hat{\varphi}^{\alpha}=\varphi^{\mu} \wedge \varphi_{\mu}{ }^{\alpha}-\frac{1}{2} \psi \wedge \theta^{\alpha}+\Phi^{\alpha}+\left(d C_{\nu}{ }^{\alpha}-C_{\mu}{ }^{\alpha} \omega_{\nu}{ }^{\mu}\right) \wedge \theta^{\nu}+i F^{\alpha} g_{\mu \bar{\nu}} \theta^{\mu} \wedge \theta^{\bar{\nu}} \quad \bmod \theta$.

On the other hand, by substituting (6.1) into the structure equation for $d \hat{\varphi}^{\alpha}$ and using Proposition 3.1 and (4.3) to replace $\hat{\varphi}^{a} \wedge \hat{\varphi}_{a}^{\alpha}$ by $\hat{D}_{\mu}^{a} \theta^{\mu} \wedge \omega_{a}^{\alpha}$ $\bmod \theta$, we obtain

$$
\begin{aligned}
d \hat{\varphi}^{\alpha}=\varphi^{\mu} \wedge & \varphi_{\mu}{ }^{\alpha}+C_{\nu}^{\mu} \theta^{\nu} \wedge \omega_{\mu}{ }^{\alpha}+\hat{D}_{\mu}{ }^{a} \theta^{\mu} \wedge \omega_{a}^{\alpha} \\
& -\frac{1}{2}\left(\psi \wedge \theta^{\alpha}+i F_{\mu} \theta^{\mu} \wedge \theta^{\alpha}-i F_{\bar{\nu}} \theta^{\bar{\nu}} \wedge \theta^{\alpha}\right)+\hat{\Phi}^{\alpha} \bmod \theta
\end{aligned}
$$

and hence, using the covariant derivative (see $\S 3$ ),

$$
\begin{aligned}
\Phi^{\alpha}+\nabla C_{\nu}{ }^{\alpha} \wedge \theta^{\nu}+ & i F^{\alpha} g_{\mu \bar{\nu}} \theta^{\mu} \wedge \theta^{\bar{\nu}}=\hat{D}_{\nu}{ }^{a} \theta^{\nu} \wedge \omega_{a}{ }^{\alpha} \\
& -\frac{1}{2}\left(i F_{\mu} \theta^{\mu} \wedge \theta^{\alpha}+i F_{\bar{\nu}} \theta^{\alpha} \wedge \theta^{\bar{\nu}}\right)+\hat{\Phi}^{\alpha} \quad \bmod \theta .
\end{aligned}
$$

Identifying coefficients in front of $\theta^{\mu} \wedge \theta^{\bar{\nu}}$ and using (3.13) we obtain:

$$
\hat{D}_{\mu}{ }^{a} \omega_{a}^{\alpha}{ }_{\bar{\nu}}-i F^{\alpha} g_{\mu \bar{\nu}}-\frac{1}{2} i F_{\bar{\nu}} \delta_{\mu}^{\alpha}=V_{\mu \bar{\nu}}^{\alpha}-\hat{V}_{\mu \bar{\nu}}^{\alpha}-C_{\mu}^{\alpha} ; \bar{\nu}
$$

or, equivalently,

$$
\hat{D}_{\mu}^{a} \omega_{a}^{\alpha \gamma}-i F^{\alpha} \delta_{\mu}^{\gamma}-\frac{1}{2} i F^{\gamma} \delta_{\mu}^{\alpha}=V_{\mu}^{\alpha}{ }^{\gamma}-\hat{V}_{\mu}^{\alpha}{ }^{\gamma}-C_{\mu}^{\alpha} ;^{\gamma}
$$

Recall that we have the symmetry relation $\omega_{\alpha}^{a}{ }_{\gamma}=\omega_{\gamma}{ }^{a} \alpha$ by (4.3) which implies $\omega_{a}^{\alpha \gamma}=\omega_{a}^{\gamma \alpha}$ in view of (4.1). We also have the symmetries $V^{\alpha}{ }_{\mu}^{\gamma}=$ $V^{\gamma}{ }_{\mu}^{\alpha}$ and $\hat{V}_{\mu}^{\gamma}{ }^{\alpha}=\hat{V}^{\alpha}{ }_{\mu}^{\gamma}$ (see [CM74, (A.14)]). By subtracting (6.13) from the same equation with $\gamma$ and $\alpha$ interchanged, we then obtain

$$
\frac{1}{2} i F^{\gamma} \delta_{\mu}^{\alpha}-\frac{1}{2} i F^{\alpha} \delta_{\mu}^{\gamma}=C_{\mu}^{\alpha} ;^{\gamma}-C_{\mu}^{\gamma} ;^{\alpha},
$$

which, after setting $\gamma=\mu$ and summing implies

$$
\frac{n-1}{2} i F^{\alpha}=C_{\mu}{ }^{\mu}{ }^{\alpha}{ }^{\alpha}-C_{\mu}{ }^{\alpha}{ }^{\mu} \text {. }
$$


Thus, for $n \geq 2$, the coefficients $F^{\alpha}$ (with respect to a fixed admissible coframe $\left.\left(\theta, \theta^{\alpha}\right)\right)$ are completely determined by the covariant derivatives of $C_{\alpha}{ }^{\beta}$ (given in terms of the uniquely defined forms $\omega_{\alpha}{ }^{\beta}$ ) and hence, in view of (6.8), by $\omega_{\alpha}{ }^{a} \beta$ and $\hat{S}_{A \bar{B} C \bar{D}}$. It now follows from the first two equations in (6.1) that the pulled back forms $\hat{\varphi}_{\alpha}{ }^{\beta}$ and $\hat{\varphi}^{\alpha}$ to $M$ are completely determined in terms of the fixed data of $M$ and the pulled back tangential pseudoconformal curvature tensor $\hat{M}$.

Next, we repeat the procedure above to $\hat{\psi}$. By differentiating the third equation in (6.1) and using the last equation of (3.12) and its analogue for $\hat{M}$ pulled back to $M$, we obtain

$$
2 i \hat{\varphi}^{\mu} \wedge \hat{\varphi}_{\mu}+2 i \hat{\varphi}^{a} \wedge \hat{\varphi}_{a}+\hat{\Psi}=2 i \varphi^{\mu} \wedge \varphi_{\mu}+\Psi+d\left(i F_{\mu} \theta^{\mu}-i F_{\bar{\nu}} \theta^{\bar{\nu}}+A \theta\right)
$$

and using again (6.1),

$$
\begin{gathered}
2 i\left(-C_{\mu}^{\gamma} \varphi_{\gamma} \wedge \theta^{\mu}+C_{\bar{\nu} \gamma} \varphi^{\gamma} \wedge \theta^{\bar{\nu}}\right)+2 i\left(C_{\mu}^{\gamma} C_{\bar{\nu} \gamma}+\hat{D}_{\mu}^{a} \hat{D}_{\bar{\nu} a}\right) \theta^{\mu} \wedge \theta^{\bar{\nu}}+\hat{\Psi} \\
=\Psi+i \nabla F_{\mu} \wedge \theta^{\mu}-i D F_{\bar{\nu}} \wedge \theta^{\bar{\nu}}+i A g_{\mu \bar{\nu}} \theta^{\mu} \wedge \theta^{\bar{\nu}} \bmod \theta
\end{gathered}
$$

By identifying the coefficients in front of $\theta^{\mu} \wedge \theta^{\bar{\nu}}$, using (3.13) and (3.19), we obtain

$$
\begin{aligned}
2 i\left(C_{\mu}^{\gamma} D_{\bar{\nu} \gamma}+C_{\bar{\nu} \gamma} D_{\mu}^{\gamma}\right)+2 i\left(C_{\mu}^{\gamma} C_{\bar{\nu} \gamma}+\hat{D}_{\mu}^{a} \hat{D}_{\bar{\nu} a}\right)-2 i \hat{P}_{\mu \bar{\nu}} \\
=-2 i P_{\mu \bar{\nu}}-i F_{\mu ; \bar{\nu}}-i F_{\bar{\nu} ; \mu}+i A g_{\mu \bar{\nu}}
\end{aligned}
$$

By contracting, we obtain

$$
n A=\left(F_{\mu ;}^{\mu}+F_{; \mu}^{\mu}\right)-2 \hat{P}_{\mu}^{\mu}+2\left(C_{\mu}^{\gamma} C_{\gamma}^{\mu}+C_{\mu}^{\gamma} D_{\gamma}^{\mu}+C_{\gamma}^{\mu} D_{\mu}^{\gamma}+\hat{D}_{\mu}^{a} \hat{D}_{a}^{\mu}\right),
$$

where we have used the trace condition $P_{\mu}{ }^{\mu}=0$.

The coefficients $D^{\mu}{ }_{\gamma}$ are given by (3.20) and hence are determined by the coframe $\left(\theta, \theta^{\alpha}\right)$. Suppose now in addition that the second fundamental form $\left(\omega_{\alpha}{ }^{a} \beta\right)$ is nondegenerate in the sense that the collection of vectors $b_{\alpha \beta}:=$ $\left(\omega_{\alpha}{ }^{n+1} \beta, \ldots, \omega_{\alpha}{ }_{\beta}^{\hat{n}}\right)$ spans $\mathbb{C}^{\hat{n}-n}$. Then $\omega_{\alpha}{ }_{\beta}{ }_{\beta}=\omega_{\alpha \bar{a} \beta}=-\omega_{\bar{a} \alpha \beta}=-\bar{\omega}_{a}{ }^{\alpha_{\bar{\beta}}}$ in view of (4.1) and hence we can solve for the coefficients $\hat{D}_{\mu}{ }^{a}$ in (6.12). Using equation (6.19), we can then determine $A$. Hence, all the pulled back forms in (6.1), as well as the forms $\hat{\varphi}_{\mu \bar{a}}=-\hat{\varphi}_{\bar{a}}$ (see the analogue of (3.19) for $\hat{M}$ ), are uniquely determined by the second fundamental form and the pulled back pseudoconformal curvature of $\hat{M}$. Now, if the manifold $\hat{M}$ is the sphere, its pseudoconformal curvature vanishes identically (see [CM74]) and the forms above are uniquely determined by the second fundamental form. We shall 
show that, when $\hat{M}$ is the sphere, all the pulled back forms $\hat{\varphi}_{A}{ }^{B}, \hat{\varphi}^{A}, \hat{\psi}$ on $M$ are determined by the second fundamental form. It remains to determine $\hat{\varphi}_{b}{ }^{a}$ and $\hat{\varphi}^{a}$ in this case. The choice of the 1 -forms $\hat{\varphi}_{b}{ }^{a}$ on $M$ is always determined up to

$$
\tilde{\hat{\varphi}}_{b}^{a}=\hat{\varphi}_{b}{ }^{a}+n_{b}{ }^{a} \theta^{\gamma}+m_{b}{ }^{a} \bar{\gamma}^{\bar{\gamma}}+p_{b}{ }^{a} \theta,
$$

where, since $\hat{\varphi}_{b \bar{a}}=-\hat{\varphi}_{\bar{a} b}$, we must have the symmetries

$$
n_{b \bar{a} \gamma}+\overline{m_{a \bar{b} \bar{\gamma}}}=p_{b \bar{a}}+\overline{p_{a \bar{b}}}=0
$$

in view of the first equation in (3.12). By the analogue of Proposition 3.1 for $\hat{M}$ and (4.3), we have

$$
\hat{\varphi}^{a}=\hat{D}_{\beta}^{a} \theta^{\beta}+\hat{E}^{a} \theta
$$

where we have used the vanishing of $\hat{\theta}^{a}$ on $M$. Since $\hat{D}_{\beta}{ }^{a}$ has already been determined, $\hat{\varphi}^{a}$ is determined up to

$$
\tilde{\hat{\varphi}}^{a}=\hat{\varphi}^{a}+G^{a} \theta
$$

for some $G^{a}$. Since every possible choice of $\hat{\varphi}_{b}{ }^{a}$ and $\hat{\varphi}^{a}$ must satisfy the structure equation (3.12) for $d \hat{\varphi}_{\beta}{ }^{a}$, we conclude, by subtracting two such possibilities from each other and using the analogue of (3.19) for $\hat{M},(4.3)$ and the vanishing of $\hat{\Phi}_{\beta}{ }^{a}$ for the sphere, that

$$
0=\left(\omega_{\beta}^{b}{ }_{\alpha} \theta^{\alpha}+\hat{D}_{\beta}^{b} \theta\right) \wedge\left(\tilde{\hat{\varphi}}_{b}^{a}-\hat{\varphi}_{b}^{a}\right)+i g_{\beta \bar{\mu}} G^{a} \theta^{\bar{\mu}} \wedge \theta .
$$

By substituting (6.20) in (6.24), we first conclude, using the nondegeneracy of $\omega_{\alpha}{ }^{a}{ }_{\beta}$, that $m_{b}{ }^{a} \bar{\beta}=0$ and, hence, by (6.21) that $n_{b}{ }^{a} \gamma=0$. Now, it easily follows from whatever is left in (6.24) that $p_{b}{ }^{a}=G^{a}=0$. This completes the proof of the following result.

Theorem 6.1. Let $f: M \rightarrow \hat{M}$ be a CR-embedding of a strictly pseudoconvex CR-manifold $M$ of dimension $2 n+1, n \geq 2$, into the unit sphere $\hat{M}=\mathbb{S}$ in $\mathbb{C}^{\hat{n}+1}$. Denote by $\left(\omega_{\alpha}{ }^{a}\right)$ the second fundamental form of $f$ relative to some coframe $\left(\theta, \theta^{A}\right)$ on $\hat{M}$ adapted to $f(M)$. If $\left(\omega_{\alpha}{ }^{a} \beta\right)$ is nondegenerate, then it uniquely determines $\left(\hat{\varphi}_{B}{ }^{A}, \hat{\varphi}^{A}, \hat{\psi}\right)$. That is, if there is another $C R$-embeddings $\tilde{f}: M \rightarrow \hat{M}$ having the same second fundamental form $\left(\omega_{\alpha}^{a}{ }_{\beta}\right)$ relative to some coframe $\left(\tilde{\theta}, \tilde{\theta}^{A}\right)$ on $\hat{M}$ adapted to $\tilde{f}(M)$ with $\tilde{f}^{*}\left(\tilde{\theta}, \tilde{\theta}^{\alpha}\right)=f^{*}\left(\theta, \theta^{\alpha}\right)$, then necessarily

$$
\tilde{f}^{*}\left(\tilde{\hat{\varphi}}_{B}{ }^{A}, \tilde{\hat{\varphi}}^{A}, \tilde{\hat{\psi}}\right)=f^{*}\left(\hat{\varphi}_{B}{ }^{A}, \hat{\varphi}^{A}, \hat{\psi}\right),
$$

where $\left(\hat{\varphi}_{B}{ }^{A}, \hat{\varphi}^{A}, \hat{\psi}\right)$ and $\left(\tilde{\hat{\varphi}}_{B}^{A}, \tilde{\hat{\varphi}}^{A}, \tilde{\hat{\psi}}\right)$ are the corresponding parts of parallellisms pulled back to $\hat{M}$. 


\section{Finitely nondegenerate mappings.}

In Theorem 6.1 above, we assumed that the second fundamental form of the embedding is nondegenerate or, equivalently, that the embedding is 2nondegenerate. This condition can be relaxed to merely requiring that the embedding is finitely nondegenerate, i.e. $k$-nondegenerate for some $k$. To do this, we first interpret $k$-nondegeneracy of the embedding as a condition on the second fundamental form that we view as a section in the vector bundle of $\mathbb{C}$-bilinear maps

$$
T_{p}^{1,0} M \times T_{p}^{1,0} M \rightarrow T_{p}^{1,0} \hat{M} / T_{p}^{1,0} M, \quad p \in M .
$$

For sections of this bundle we have the covariant differential induced by the pseudohermitian connections $\nabla$ and $\hat{\nabla}$ on $M$ and $\hat{M}$ respectively:

$$
\nabla \omega_{\alpha \beta}^{a}=d \omega_{\alpha \beta}^{a}-\omega_{\mu}^{a}{ }_{\beta} \hat{\omega}_{\alpha}^{\mu}+\omega_{\alpha \beta}^{b} \hat{\omega}_{b}^{a}-\omega_{\alpha}^{a}{ }_{\mu} \hat{\omega}_{\beta}^{\mu}
$$

As above, we also use e.g. $\omega_{\alpha}{ }_{\beta ; \gamma}$ to denote its component in the direction $\theta^{\gamma}$. Higher order covariant derivatives $\omega_{\alpha}^{a} \beta ; \gamma_{1}, \ldots, \gamma_{l}$ are defined inductively in a similar way:

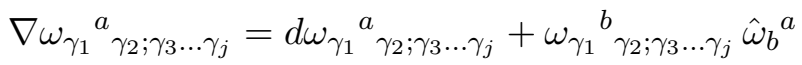

$$
\begin{aligned}
& -\sum_{l=1}^{j} \omega_{\gamma_{1}}^{a} \gamma_{2} ; \gamma_{3} \ldots \gamma_{l-1} \mu \gamma_{l+1} \ldots \gamma_{j} \hat{\omega}_{\gamma_{l}}{ }^{\mu},
\end{aligned}
$$

To obtain the desired relation between finite nondegeneracy and covariant derivatives of $\omega_{\alpha}{ }^{a} \beta$, we begin by applying $L_{\gamma}$ to (4.6) and using the analogue of (3.6) for $\hat{M}$ :

$\left.\left.L_{\gamma} L_{\alpha} L_{\beta}\left(\hat{\rho}_{\bar{Z}^{\prime}} \circ f\right)=L_{\gamma}\right\lrcorner d\left(\omega_{\alpha \bar{a} \beta} \theta^{\bar{a}}\right)=L_{\gamma}\right\lrcorner\left(d \omega_{\alpha \bar{a} \beta} \wedge \theta^{\bar{a}}-\omega_{\alpha \bar{a} \beta} \hat{\omega}_{\bar{b}}^{\bar{a}} \wedge \theta^{\bar{b}}\right) \quad \bmod \theta, \theta^{\bar{\alpha}}$.

We now observe that the expression in the brackets on the right hand side coincides with $\nabla \omega_{\alpha \bar{a} \beta} \wedge \theta^{\bar{a}}$ modulo $\bar{E}_{2}$ (as defined in $\S 2$ ) which is spanned by the conjugated forms (4.8). Hence we obtain

$$
L_{\gamma} L_{\alpha} L_{\beta}\left(\hat{\rho}_{\bar{Z}^{\prime}} \circ f\right)=\omega_{\alpha \bar{a} \beta ; \gamma} \theta^{\bar{a}} \bmod \bar{E}_{2}
$$

and, by induction,

$$
L_{\gamma_{l}} \ldots L_{\gamma_{1}} L_{\alpha} L_{\beta}\left(\hat{\rho}_{\bar{Z}^{\prime}} \circ f\right)=\omega_{\alpha \bar{a} \beta ; \gamma_{1}, \ldots, \gamma_{l}} \theta^{\bar{a}} \bmod \bar{E}_{l+1}
$$


for any integer $l>1$, where $E_{l+1}$ is as defined in $\S 2$ and is spanned by

$$
\theta, \theta^{\bar{a}}, \omega_{\alpha \bar{a} \beta ; \gamma_{1}, \ldots, \gamma_{s}} \theta^{\bar{a}}, \quad 0 \leq s \leq l-1 .
$$

Hence, we obtain the following generalization of the equivalence of 2nondegeneracy with nondegeneracy of the second fundamental form:

Proposition 7.1. For $k_{0} \geq 2$, the embedding $f: M \rightarrow \hat{M}$ is $k_{0}$ nondegenerate at a point $p$ if and only if the equality

$$
\operatorname{span}\left\{\omega_{\gamma_{1}}{ }^{a} \gamma_{2} ; \gamma_{3}, \ldots, \gamma_{l} L_{a}, 2 \leq l \leq k\right\}(p)=N_{f(p)} M
$$

holds for $k \geq k_{0}$ but not for $k<k_{0}$. Furthermore, $f$ is $\left(k_{0}, s_{0}\right)$-nondegenerate at $p$ if and only if the codimension of the span on the left hand side in (7.5) in the normal space $N_{f(p)} M$ is equal to $s_{0}$ for $k \geq k_{0}$ and strictly smaller for $k<k_{0}$.

We shall now prove the following alternative to Theorem 6.1. (The reader should note that Theorem 7.2 is more general than Theorem 6.1 in that it allows for a higher degree $k$ of nondegeneracy, but is a little weaker than Theorem 6.1 in the special case $k=2$, i.e. in the case of nondegenerate second fundamental form, since the theorem below requires also the covariant derivatives $\omega_{\alpha}^{a} \beta ; \gamma$ to determine the induced pseudoconformal connection.)

Theorem 7.2. Let $f: M \rightarrow \hat{M}$ be a $k$-nondegenerate $C R$-embedding of a strictly pseudoconvex $C R$-manifold $M$ of dimension $2 n+1, n \geq 2$, into the unit sphere $\hat{M}=\mathbb{S}$ in $\mathbb{C}^{\hat{n}+1}$. Denote by $\left(\omega_{\alpha}{ }^{a}{ }_{\beta}\right)$ the second fundamental form of $f$ relative to some coframe $\left(\theta, \theta^{A}\right)$ on $\hat{M}$ adapted to $f(M)$. Then the covariant derivatives $\left(\omega_{\alpha}{ }^{a} \beta ; \gamma_{1} \ldots, \gamma_{j}\right)_{0 \leq j \leq k-1}$ uniquely determine $\left(\hat{\varphi}_{B}{ }^{A}, \hat{\varphi}^{A}, \hat{\psi}\right)$. That is, if $\tilde{f}: M \rightarrow \hat{M}$ is another $C R$-embedding whose second fundamental form with respect to a coframe $\left(\tilde{\theta}, \tilde{\theta}^{A}\right)$ on $\hat{M}$ adapted to $\tilde{f}(M)$ with $\tilde{f}^{*}\left(\tilde{\theta}, \tilde{\theta}^{\alpha}\right)=f^{*}\left(\theta, \theta^{\alpha}\right)$ is denoted by $\tilde{\omega}_{\alpha}{ }^{a}{ }_{\beta}$ then

$$
\omega_{\alpha}^{a}{ }_{\beta ; \gamma_{1} \ldots \gamma_{j}}=\tilde{\omega}_{\alpha}{ }^{a}{ }_{\beta ; \gamma_{1} \ldots \gamma_{j}}, \quad 0 \leq j \leq k-1,
$$

implies $\tilde{f}^{*}\left(\tilde{\hat{\varphi}}_{B}^{A}, \tilde{\hat{\varphi}}^{A}, \tilde{\hat{\psi}}\right)=f^{*}\left(\hat{\varphi}_{B}{ }^{A}, \hat{\varphi}^{A}, \hat{\psi}\right)$.

Proof. Let us for brevity use the notation $\left(\hat{\varphi}_{B}{ }^{A}, \hat{\varphi}^{A}, \hat{\psi}\right)$ and $\left(\tilde{\hat{\varphi}}_{B}{ }^{A}, \tilde{\hat{\varphi}}^{A}, \tilde{\hat{\psi}}\right)$ also for the pullbacks to $M$. If we examine the arguments above leading to Theorem 6.1 , we see that the nondegeneracy of the second fundamental form 
has not been used to obtain $\left(\tilde{\hat{\varphi}}_{\beta}^{\alpha}, \tilde{\hat{\varphi}}^{\alpha}\right)=\left(\hat{\varphi}_{\beta}{ }^{\alpha}, \hat{\varphi}^{\alpha}\right)$. To compare the normal components $\hat{\varphi}_{b}{ }^{a}$ and $\tilde{\hat{\varphi}}_{b}{ }^{a}$ we write as before the general relation between them in the form (6.20-6.21). In view of (7.2), the analogues of (3.19) for $\hat{M}$ and the fact that $\hat{\omega}_{\beta}{ }^{\alpha}=\omega_{\beta}{ }^{\alpha}$ (see $\S 4$ ), the equations (7.6) imply that

$$
\omega_{\gamma_{1}}^{b} \gamma_{2} ; \gamma_{3} \ldots \gamma_{j} n_{b}^{a}{ }_{\gamma}=0, \quad 2 \leq j \leq k .
$$

Since we assume $f$ to be $k$-nondegenerate, we conclude from Proposition 7.1 that $n_{b}{ }_{\gamma}{ }_{\gamma}=0$ and hence, by (6.21), also $m_{b}{ }^{a} \bar{\gamma}=0$. It follows that

$$
\tilde{\hat{\varphi}}_{b}{ }^{a}=\hat{\varphi}_{b}{ }^{a}+p_{b}{ }^{a} \theta
$$

which, in turn, implies $\tilde{\hat{\omega}}_{b}^{a}=\hat{\omega}_{b}^{a} \bmod \theta$ and therefore

$$
\omega_{\alpha}^{a}{ }_{\beta ; \bar{\gamma}}=\tilde{\omega}_{\alpha \beta}^{a}{ }_{\beta ;} .
$$

By the analogue of (3.19) for $\hat{M}$ and (4.3),

$$
\omega_{\alpha}^{a}{ }_{\beta} \theta^{\beta}+\hat{D}_{\alpha}^{a} \theta=\hat{\varphi}_{\alpha}^{a},
$$

and similarly for $\tilde{\hat{\varphi}}_{\alpha}{ }^{a}$. We claim that $\hat{D}_{\alpha}^{a}=\tilde{\hat{D}}_{\alpha}{ }^{a}$. To see this, we differentiate (7.10), use the fourth identity in (3.12) for $d \hat{\varphi}_{\alpha}{ }^{a}$ and identify the coefficients of $\theta^{\beta} \wedge \theta^{\bar{\gamma}}$ :

$$
\omega_{\alpha}^{a} \beta ; \bar{\gamma}=i\left(g_{\alpha \bar{\gamma}} \hat{D}_{\beta}^{a}+g_{\beta \bar{\gamma}} \hat{D}_{\alpha}^{a}\right)
$$

whose contraction yields

$$
\omega_{\mu}^{a}{ }_{\beta}^{\mu}=i(n+1) \hat{D}_{\beta}^{a} .
$$

Thus, $\hat{D}_{\alpha}{ }^{a}$ is uniquely determined by $\omega_{\alpha}{ }^{a} \beta \bar{\gamma}$ proving the claim in view of (7.9). Then $\tilde{\hat{\varphi}}_{\alpha}{ }^{a}=\hat{\varphi}_{\alpha}{ }^{a}$ by (7.10) and the analogue of (3.19) for $\hat{M}$ implies in view of (4.3) that $\tilde{\hat{\varphi}}^{a}$ and $\hat{\varphi}^{a}$ are related by (6.23) with suitable $G^{a}$. Moreover, it follows from equation (6.19) that $A=\tilde{A}$ and hence $\hat{\psi}=\tilde{\hat{\psi}}$.

To finish the proof, we must show that $p_{a}{ }^{b}=G^{a}=0$. For this, we rewrite (6.24)

$$
0=\omega_{\alpha}^{b}{ }_{\beta} p_{b}^{a} \theta^{\alpha} \wedge \theta+i g_{\beta \bar{\nu}} G^{a} \theta^{\bar{\nu}} \wedge \theta
$$

from which it follows that $G^{a}=0$ (and $\omega_{\alpha}{ }^{a}{ } p_{a}{ }^{b}=0$, which does not necessarily imply $p_{a}{ }^{b}=0$ unless the second fundamental form is nondegenerate). Hence $\hat{\varphi}^{a}=\tilde{\hat{\varphi}}^{a}$. To conclude that $p_{a}{ }^{b}=0$, we differentiate the equation (7.8), use the structure equations for $\hat{\varphi}_{a}{ }^{b}$ and $\tilde{\hat{\varphi}}_{a}{ }^{b}$ and identify the coefficient 
in front of $\theta^{\alpha} \wedge \theta^{\bar{\beta}}$ that yields $i g_{\alpha \bar{\beta}} p_{a}{ }^{b}=0$ and hence clearly implies $p_{a}{ }^{b}=0$. The proof of Theorem 7.2 is complete.

Theorem 7.2 implies that the induced pseudoconformal connection is determined by the second fundamental form and its covariant derivatives with respect to this connection. We shall now prove these covariant derivatives, in turn, are controlled by the (pseudoconformal) Gauss equation. The result is the following.

Theorem 7.3. Let $f: M \rightarrow \hat{M}$ be a CR-embedding of a strictly pseudoconvex CR manifold of dimension $2 n+1$ into the unit sphere $\hat{M}=\mathbb{S}$ in $\mathbb{C}^{\hat{n}+1}$ with $\hat{n}-n<n / 2$. Denote by $\left(\omega_{\alpha}{ }^{a} \beta\right)$ the second fundamental form of $f$ relative to a coframe $\left(\theta, \theta^{A}\right)$ on $\hat{M}$ adapted to $f(M)$ and by $\omega_{\alpha}{ }^{a}{ }_{\beta ; \gamma_{1}, \ldots, \gamma_{l}}$ its covariant derivatives (with respect to the pseudohermitian structures on $M$ and $\hat{M}$ ). Fix an integer $k \geq 2$ such that the space $E_{k}=E_{k}(p)$ (spanned by (7.4)) is of constant dimension for $p \in M$. Then the collection of derivatives $\omega_{\gamma_{1}}{ }^{a} \gamma_{2} ; \gamma_{3}, \ldots, \gamma_{l}$ for $2 \leq l \leq k$ is uniquely determined by $\left(\theta, \theta^{\alpha}\right)$ up to unitary transformations of $\mathbb{C}^{\hat{n}-n}$, i.e. for any other CR-embedding $\tilde{f}: M \rightarrow \hat{M}$ and any coframe $\left(\tilde{\theta}, \tilde{\theta}^{A}\right)$ on $\hat{M}$ adapted to $\tilde{f}(M)$ with $\tilde{f}^{*}\left(\tilde{\theta}, \tilde{\theta}^{\alpha}\right)=f^{*}\left(\theta, \theta^{\alpha}\right)$, one has

$$
\tilde{\omega}_{\gamma_{1}}{ }^{a} \gamma_{2} ; \gamma_{3}, \ldots, \gamma_{l}=\omega_{\gamma_{1}}{ }^{a} \gamma_{2} ; \gamma_{3}, \ldots, \gamma_{l}, \quad 2 \leq l \leq k,
$$

after a possible unitary change of $\left(\tilde{\theta}^{a}\right)$ (near any given point).

Proof. As in the proof of Lemma 5.3 and Corollary 5.5, it is sufficient to prove that the collections of vectors

$$
\left(\omega_{\gamma_{1}}{ }^{a} \gamma_{2} ; \gamma_{3}, \ldots, \gamma_{l} L_{a}\right)_{2 \leq l \leq k} \quad \text { and } \quad\left(\tilde{\omega}_{\gamma_{1}}{ }^{a} \gamma_{2} ; \gamma_{3}, \ldots, \gamma_{l} \tilde{L}_{a}\right)_{2 \leq l \leq k}
$$

have the same scalar products with respect to $\left(g_{a \bar{b}}\right)$ (where $\left(T, L_{A}\right)$ and $\left(\tilde{T}, \tilde{L}_{A}\right)$ are dual frames to $\left(\theta, \theta^{A}\right)$ and $\left(\tilde{\theta}, \tilde{\theta}^{A}\right)$ respectively), i.e. that

$g_{a \bar{b}} \omega_{\gamma_{1}}{ }^{a} \gamma_{2} ; \gamma_{3}, \ldots, \gamma_{l} \omega_{\bar{\delta}_{1}} \bar{b}_{\delta_{2} ; \bar{\delta}_{3}, \ldots, \bar{\delta}_{s}}=g_{a \bar{b}} \tilde{\omega}_{\gamma_{1}}{ }^{a} \gamma_{2} ; \gamma_{3}, \ldots, \gamma_{l} \tilde{\omega}_{\bar{\delta}_{1}} \bar{b}_{\bar{\delta}_{2} ; \bar{\delta}_{3}, \ldots, \bar{\delta}_{s}}, \quad 2 \leq l, s \leq k$,

(the constant dimension assumption on $E_{k}$ is needed to guarantee that that the unitary change of $\left(\theta^{a}\right)$ can be made smooth; otherwise a nonsmooth change is still possible). For this, we observe that $\hat{S}_{\alpha \bar{\beta}} \mu \bar{\nu}=0$ since $\hat{M}$ is the unit sphere and rewrite the pseudoconformal Gauss equation (5.7) in this case as

$$
0=S_{\alpha \bar{\beta} \mu \bar{\nu}}+g_{a \bar{b}} \omega_{\alpha}^{a}{ }_{\mu} \omega_{\bar{\beta}}^{\bar{b}} \bar{\nu}+T_{\alpha \bar{\beta} \mu \bar{\nu}}
$$


where $T_{\alpha \bar{\beta}} \mu \bar{\nu}$ is a tensor conformally equivalent to 0 (see $\S 3$ ), i.e. a linear combination of $g_{\alpha \bar{\beta}}, g_{\alpha \bar{\nu}}, g_{\mu \bar{\beta}}$ and $g_{\mu \bar{\nu}}$. More generally, we call a tensor $T_{\alpha_{1}, \ldots, \alpha_{r}, \bar{\beta}_{1}, \ldots, \bar{\beta}_{s}}^{a_{1} \ldots a_{t} \bar{b}_{1} \ldots \bar{b}_{q}}$ (and similar tensors with different order of indices) conformally equivalent to 0 or conformally flat if it is a linear combination of $g_{\alpha_{i} \bar{\beta}_{j}}$ for $i=1, \ldots, r, j=1, \ldots, s$.

We will show (7.16) inductively by comparing covariant derivatives of the right hand side in $(7.17)$ and in its analogue for $\left(\tilde{\theta}, \tilde{\theta}^{\alpha}\right)$. When calculating the derivatives, the following two remarks with be of importance. First, since we have assumed the Levi form matrix $\left(g_{\alpha \bar{\beta}}\right)$ to be constant, it is easy to see that covariant derivatives of conformally flat tensors (with respect to any connection) are always conformally flat. Second, in view of (7.11), the "mixed" derivatives $\omega_{\alpha}^{a} \beta ; \bar{\gamma}$ are conformally flat together with all their higher order derivatives. Finally, in order to treat e.g. derivatives $\omega_{\alpha}{ }^{a}{ }_{\beta ; \gamma \bar{\delta}}$ we shall need the following lemma, which describes how covariant derivatives commute.

Lemma 7.4. In the setting of Theorem 7.3, for any $s \geq 2$, we have a relation

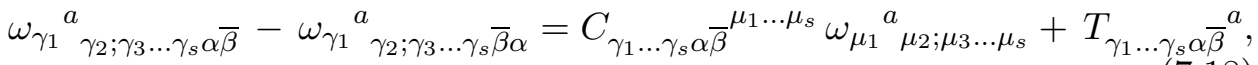

where the tensor $C_{\gamma_{1} \ldots \gamma_{s} \alpha \bar{\beta}}{ }^{\mu_{1} \ldots \mu_{s}}$ depends only on $\left(\theta, \theta^{\alpha}\right)$ (and not on the embedding $f$ ) and $T_{\gamma_{1} \ldots \gamma_{s} \alpha \bar{\beta}}{ }^{a}$ is conformally flat.

Proof. By observing that the left hand side of the identity (7.18) is a tensor, it is enough to show, for each fixed point $p \in M$, the identity at $p$ with respect to any particular choice of $\left(\theta, \theta^{A}\right)$ depending smoothly on $p$. By making a suitable unitary change of coframe $\theta^{\alpha} \rightarrow u_{\beta}^{\alpha} \theta^{\beta}$ and $\theta^{a} \rightarrow u_{b}{ }^{a} \theta^{b}$ (in the tangential and normal directions respectively), we may chose a coframe smoothly depending on $p$ with $\hat{\omega}_{\alpha}^{\beta}(p)=\hat{\omega}_{a}^{b}(p)=0$. Relative to such a coframe, the left hand side of (7.18) evaluated at $p$ equals, in view of (7.2) and (3.19), modulo a conformally flat tensor, to the coefficient in front of $\theta^{\alpha} \wedge \theta^{\bar{\beta}}$ in the expression

$$
\sum_{j=1}^{s} \omega_{\gamma_{1}}^{a}{ }_{\gamma_{2} ; \gamma_{3} \ldots \gamma_{j-1}}^{\mu \gamma_{j+1} \gamma_{s}} d \omega_{\gamma_{j}}{ }^{\mu}-\omega_{\gamma_{1}}{ }^{b} \gamma_{2} ; \gamma_{3} \ldots \gamma_{s} d \hat{\varphi}_{b}{ }^{a},
$$

where we have used that $\hat{\omega}_{\alpha}^{\beta}=\omega_{\alpha}^{\beta}$ (see $\S 4$ ). Next, we observe, by examining the equations (6.1) and (6.8), that the coefficients in front of $\theta^{\alpha}$ and $\theta^{\bar{\beta}}$ in the pulled back forms $\hat{\varphi}^{\gamma}$ are uniquely determined by $\left(\theta, \theta^{\alpha}\right)$ and the scalar 
products $g_{a \bar{b}} \omega_{\alpha}{ }_{\mu}{ }_{\mu} \omega_{\bar{\beta}} \bar{b} \bar{\nu}$. The latter are, in turn, uniquely determined by $\left(\theta, \theta^{\alpha}\right)$ in view of Lemma 5.3 (ii) (applied to (7.17)). We then compute $d \hat{\varphi}_{b}{ }^{a}$ using the structure equation and the vanishing of $\theta^{a}$ on $M$ and of $\hat{\varphi}_{\beta}{ }^{\alpha}$ and $\hat{\varphi}_{b}{ }^{a}$ at $p$ modulo $\theta$ :

$$
d \hat{\varphi}_{b}{ }^{a}=\hat{\varphi}_{b}{ }^{\mu} \wedge \hat{\varphi}_{\mu}{ }^{a}-i \delta_{b}{ }^{a} \hat{\varphi}_{\mu} \wedge \theta^{\mu} \bmod \theta .
$$

In view of $(4.1-4.3)$ and $(3.19)$, the first term on the right hand side does not contribute to the the coefficient in front of $\theta^{\alpha} \wedge \theta^{\bar{\beta}}$. Hence we conclude that the coefficient in front of $\theta^{\alpha} \wedge \theta^{\bar{\beta}}$ in (7.19), at $p$, is of the required form. This completes the proof of Lemma 7.4.

We now return to the proof of Theorem 7.3 and show the required indentities (7.16) by induction on the number of indices $l+s$. The case $l+s=4$ (i.e. $l=s=2$ ) follows from Lemma 5.3 (ii) applied to (7.17). Since we have chosen coframes for which the Levi form $g_{A \bar{B}}$ is constant, we have

$$
\hat{\nabla} g_{A \bar{B}}=d g_{A \bar{B}}-g_{C \bar{B}} \hat{\omega}_{A}^{C}-g_{A \bar{D}} \hat{\omega}_{\bar{B}}{ }^{\bar{D}}=-\left(\hat{\omega}_{A \bar{B}}+\hat{\omega}_{\bar{B} A}\right)=0 .
$$

In order to show (7.16) for any $2 \leq l, s \leq k$ with $l+s>2$ we covariantly differentiate (7.17) for $\alpha=\gamma_{1}, \mu=\gamma_{2}, \beta=\delta_{1}, \nu=\delta_{2}$ with respect to the indices $\gamma_{3}, \ldots, \gamma_{l}$ and $\bar{\delta}_{3}, \ldots, \bar{\delta}_{s}$ consequently. We obtain that the left hand side in (7.16) is uniquely determined by the covariant derivatives of $S_{\alpha \bar{\beta}} \mu \bar{\nu}$ modulo a conformally flat tensor and modulo other scalar product terms involving either derivatives of $\omega_{\gamma_{1}}{ }^{a} \gamma_{2}$ with respect to indices one of which is $\delta_{j}$ or derivatives of $\omega_{\bar{\delta}_{1}} \bar{b}_{\bar{\delta}_{2}}$ with respect to indices one of which is $\gamma_{j}$. If such an index appears at the beginning, the corresponding scalar product is conformally flat in view of (7.11). Otherwise, by interchanging indices and applying Lemma 7.4, we see that the scalar product term is equal, again modulo a conformally flat tensor, to a sum of scalar products of the expressions on the right hand side of (7.18) and its derivatives. These scalar products are linear combinations of the expressions on the left hand side in (7.16) with a smaller number of indices $l+k$. Hence, by the induction, they are uniquely determined by $\left(\theta, \theta^{\alpha}\right)$. Summarizing, we obtain the equality in (7.16) modulo a conformally flat tensor $T_{\gamma_{1}, \ldots, \gamma_{l} \bar{\delta}_{1} \ldots, \bar{\delta}_{s}}$. The proof of Theorem 7.3 is completed by applying [Hu99, Lemma 3.2] as in the proof of Lemma 5.3 to conclude that $T_{\gamma_{1}, \ldots, \gamma_{l} \bar{\delta}_{1} \ldots, \bar{\delta}_{s}}=0$.

\section{Adapted $Q$-frames and proof of Theorem 2.1 in case $s=0$.}

We embed $\mathbb{C}^{\hat{n}+1}$ in projective space $\mathbb{P}^{\hat{n}+1}$ in the usual way, i.e. as the set $\left\{\zeta^{0} \neq 0\right\}$ in the homogeneous coordinates $\left[\zeta^{0}: \zeta^{1}: \ldots: \zeta^{\hat{n}+1}\right]$, and, following 
$[\mathrm{CM} 74, \S 1]$, realize the unit sphere as the quadric $Q$ given in $\mathbb{P}^{\hat{n}+1}$ by the equation $(\zeta, \zeta)=0$, where the Hermitian scalar product $(\cdot, \cdot)$ is defined by

$$
(\zeta, \tau):=\delta_{A \bar{B}} \zeta^{A} \overline{\tau^{B}}+\frac{i}{2}\left(\zeta^{\hat{n}+1} \overline{\tau^{0}}-\zeta^{0} \overline{\tau^{\hat{n}+1}}\right)
$$

Recall here the index convention from previous sections: capital Latin indices run from 1 to $\hat{n}$. A $Q$-frame (see e.g. [CM74]) is a unimodular basis $\left(Z_{0}, Z_{1}, \ldots, Z_{\hat{n}+1}\right)$ of $\mathbb{C}^{\hat{n}+2}$ (i.e. $\left.\operatorname{det}\left(Z_{0}, \ldots, Z_{\hat{n}+1}\right)=1\right)$ such that $Z_{0}$ and $Z_{\hat{n}+1}$, as points in $\mathbb{P}^{\hat{n}+1}$, are on $Q$, the vectors $\left(Z_{A}\right)$ form an orthonormal basis (relative to the inner product (8.1)) for the complex tangent space to $S$ at $Z_{0}$ and $Z_{\hat{n}+1}$ and $\left(Z_{\hat{n}+1}, Z_{0}\right)=i / 2$. (We shall denote the corresponding points in $\mathbb{P}^{\hat{n}+1}$ also by $Z_{0}$ and $Z_{\hat{n}+1}$; it should be clear from the context whether the point is in $\mathbb{C}^{\hat{n}+2}$ or $\mathbb{P}^{\hat{n}+1}$.) Equivalently, a $Q$-frame is any unimodular basis satisfying

$$
\left(Z_{A}, Z_{A}\right)=1, \quad\left(Z_{\hat{n}+1}, Z_{0}\right)=-\left(Z_{0}, Z_{\hat{n}+1}\right)=i / 2,
$$

while all other scalar products are zero.

On the space $\mathcal{B}$ of all $Q$-frames there is a natural free transitive action of the group $\operatorname{SU}(\hat{n}+1,1)$ of unimodular $(\hat{n}+2) \times(\hat{n}+2)$ matrices that preserve the inner product (8.1). Hence, any fixed $S$-frame defines an isomorphism between $\mathcal{B}$ and $\operatorname{SU}(\hat{n}+1,1)$. On the space $\mathcal{B}$, there are Maurer-Cartan forms $\pi_{\Lambda}{ }^{\Omega}$, where capital Greek indices run from 0 to $\hat{n}+1$, defined by

$$
d Z_{\Lambda}=\pi_{\Lambda}^{\Omega} Z_{\Omega}
$$

and satisfying $d \pi_{\Lambda}{ }^{\Omega}=\pi_{\Lambda}{ }^{\Gamma} \wedge \pi_{\Gamma}{ }^{\Omega}$. Here the natural $\mathbb{C}^{\hat{n}+2}$-valued 1-forms $d Z_{\Lambda}$ on $\mathcal{B}$ are defined as differentials of the map $\left(Z_{0}, \ldots, Z_{\hat{n}+2}\right) \mapsto Z_{\Lambda}$.

Recall [CM74, W79] that a smoothly varying $Q$-frame $\left(Z_{\Lambda}\right)=\left(Z_{\Lambda}(p)\right)$ for $p \in Q$ is said to be adapted to $Q$ if $Z_{0}(p)=p$ as points of $\mathbb{P}^{\hat{n}+1}$. It is shown in $[\mathrm{CM} 74, \S 5]$ that, if we use an adapted $Q$-frame to pull back the 1-forms $\pi_{\Lambda}{ }^{\Omega}$ from $\mathcal{B}$ to $Q$ and set

$$
\theta:=\frac{1}{2} \pi_{0}^{\hat{n}+1}, \quad \theta^{A}:=\pi_{0}{ }^{A}, \quad \xi:=-\pi_{0}^{0}+\overline{\pi_{0}^{0}},
$$

we obtain a coframe $\left(\theta, \theta^{A}\right)$ on $Q$ and a form $\xi$ satisfying the structure equation

$$
d \theta=i \delta_{A \bar{B}} \theta^{A} \wedge \theta^{\bar{B}}+\theta \wedge \xi .
$$

In particular, it follows from (8.3) that the coframe $\left(\theta^{\alpha}, 2 \theta\right)$ is dual to the frame defined by $\left(Z_{A}, Z_{\hat{n}+}\right)$ on $M$ and hence depends only on the values 
of $\left(Z_{\Lambda}\right)$ at the same points. Furthermore, there exists a unique section $M \rightarrow Y$ for which the pullbacks of the forms $\left(\omega, \omega^{\alpha}, \varphi\right)$ in $(3.11)$ are $\left(\theta, \theta^{\alpha}, \xi\right)$ respectively. Then the pulled back forms $\left(\hat{\varphi}_{B}{ }^{A}, \hat{\varphi}^{A}, \hat{\psi}\right)$ are given by [CM74, $(5.8 \mathrm{~b})]$

$$
\hat{\varphi}_{B}{ }^{A}=\pi_{B}{ }^{A}-\delta_{B}{ }^{A} \pi_{0}^{0}, \quad \hat{\varphi}^{A}=2 \pi_{\hat{n}+1}{ }^{A}, \quad \hat{\psi}=-4 \pi_{\hat{n}+1}^{0} .
$$

As in $[\mathrm{CM} 74,(5.30)]$, the pulled back forms $\pi_{\Lambda}{ }^{\Omega}$ can be uniquely solved from $(8.3-8.4)$ :

$$
\begin{aligned}
& (\hat{n}+2) \pi_{0}{ }^{0}=-\hat{\varphi}_{C}{ }^{C}-\xi, \quad \pi_{0}{ }^{A}=\theta^{A}, \quad \pi_{0}{ }^{\hat{n}+1}=2 \theta, \\
& \pi_{A}{ }^{0}=-i \hat{\varphi}_{A}, \quad \pi_{A}{ }^{B}=\hat{\varphi}_{A}{ }^{B}+\delta_{A}{ }^{B} \pi_{0}{ }^{0}, \quad \pi_{A}^{\hat{n}+1}=2 i \theta_{A}, \\
& 4 \pi_{\hat{n}+1}{ }^{0}=-\hat{\psi}, \quad 2 \pi_{\hat{n}+1}^{A}=\hat{\varphi}^{A}, \quad(\hat{n}+2) \pi_{\hat{n}+1}{ }^{\hat{n}+1}=\hat{\varphi}_{\bar{D}} \bar{D}+\xi .
\end{aligned}
$$

Thus, the pullback of $\pi_{\Lambda} \Omega$ is completely determined by the pullbacks $\left(\theta, \theta^{A}, \xi, \hat{\varphi}_{B}{ }^{A}, \hat{\varphi}^{A}, \hat{\psi}\right)$.

In an adapted $Q$-frame $\left(Z_{\Lambda}\right)$, the vector $Z_{0}$ giving the referenc point is determined up to a factor $t \in \mathbb{C}$ and a change $\left(Z_{0}, Z_{A}, Z_{\hat{n}+1}\right) \mapsto$ $\left(t Z_{0}, Z_{A}, t^{-1} Z_{\hat{n}+1}\right)$ results in the change $\theta \mapsto|t|^{2} \theta$ (see [CM74, (5.10)]). Following [CM74], denote by $H_{1}$ the subgroup of $\mathrm{SU}(\hat{n}+1,1)$ fixing the point $Z_{0}$ in $\mathbb{P}^{\hat{n}+1}$ and the length of $Z_{0}$ in $\mathbb{C}^{\hat{n}+2}$. Then, there is a surjective homomorphism $H_{1} \rightarrow G_{1}$, explicitly described in [CM74, (5.12)], onto the group $G_{1}$ acting transitively on the coframes $\left(\omega, \omega^{A}, \omega^{\bar{A}}, \varphi\right)$ (on the bundle $E$ where $\omega$ is fixed) such that the relations (8.5) are preserved. We conclude, in particular, that for any choice of an admissible coframe $\left(\theta, \theta^{A}\right)$ on $Q$ (as defined in $\S 3)$, there exists an adapted $Q$-frame $\left(Z_{\Lambda}\right)$ such that (8.5) holds with $\xi=0$.

Proof of Theorem 2.1 in case $s=0$. Let $f, \tilde{f}: M \rightarrow \mathbb{S}$ be two CRembeddings as in Theorem 1.2 and let $p \in M$ be a point where $M$ is $k_{0}$-nondegenerate. Then the space $E_{k_{0}}(q)$ defined by $(2.1)$ is of maximal and hence constant dimension for $q \in M$ near $p$. Choose any admissible coframe $\left(\theta, \theta^{\alpha}\right)$ on $M$ near $p$ (in the terminology of $\S 3$ ). Then Corollary 4.2 applied to $f$ and $\tilde{f}$ yields admissible coframes $\left(\hat{\theta}, \hat{\theta}^{A}\right)$ and $\left(\tilde{\hat{\theta}}, \hat{\hat{\theta}}^{A}\right)$ on $\mathbb{S}$ near $f(p)$ and $\tilde{f}(p)$ respectively that are both adapted to $\left(\theta, \theta^{\alpha}\right)$ as defined after Corollary 4.2. Hence, by Theorem 7.3, there exists a further unitary change of the coframe $\left(\tilde{\hat{\theta}}, \tilde{\hat{\theta}}^{A}\right)$ near $\tilde{f}(p)$ such that one has the equality (7.14) for the covariant derivatives up to order $k_{0}-2$ of the second fundamental forms of $f$ and $\tilde{f}$. Now Theorem 7.2 can be applied to conclude that the connection 
forms $\left(\hat{\varphi}_{B}{ }^{A}, \hat{\varphi}^{A}, \hat{\psi}\right)$ and $\left(\tilde{\hat{\varphi}}_{B}{ }^{A}, \tilde{\hat{\varphi}}^{A}, \tilde{\hat{\psi}}\right)$ coincide when pulled back via $f$ and $\tilde{f}$ respectively.

As described above we realize the sphere $\mathbb{S}$ as the quadric $Q$ and construct two adapted $Q$-frames $\left(Z_{\Lambda}\right)$ and $\left(\tilde{Z}_{\Lambda}\right)$ on $Q$ near $f(p)$ and $\tilde{f}(p)$ respectively such that the relations $(8.5)$ and their analogues for $\tilde{f}$ hold. We conlcude that also the corresponding Maurer-Cartan forms $\left(\pi_{\Lambda}{ }^{\Omega}\right)$ and $\left(\tilde{\pi}_{\Lambda}{ }^{\Omega}\right)$ coincide when pulled back to $M$ via $f$ and $\tilde{f}$ respectively.

The end of the proof follows the line of [W78, Lemma (1.1)]. Since the group $\mathrm{SU}(\hat{n}+1,1)$ acts transitively on the space of all $Q$-frames, we can compose $\tilde{f}$ with such a transformation to obtain $Z_{\Lambda}(f(p))=\tilde{Z}_{\Lambda}(\tilde{f}(p))$. Now the relation $f^{*} \pi_{\Lambda}{ }^{\Omega}=\tilde{f}^{*} \tilde{\pi}_{\Lambda}{ }^{\Omega}$ implies that, along any sufficiently small real curve in $M$, starting from $p$, both $Q$-frames $Z_{\Lambda}(f(q))$ and $\tilde{Z}_{\Lambda}(\tilde{f}(q))$ satisfy the same first order ODE with the same initial values at $p$. Hence we must have $Z_{\Lambda}(f(q))=\tilde{Z}_{\Lambda}(\tilde{f}(q))$ for $q \in M$ near $p$ and therefore $f(q)=\tilde{f}(q)$ since $Z_{0}(f(q))$ and $\tilde{Z}_{0}(f(q))$ give, as points in $\mathbb{P}^{\hat{n}+1}$, exactly the reference points by the definition of the adapted frames. Finally, the global coincidence $f \equiv \tilde{f}$ follows from the local one by the well known facts that any CR-manifold of hypersurface type that is embeddable into a sphere is automatically minimal and that that two CR-functions on a minimal connected CR-manifold coincide if and only if they coincide in a nonempty open subset.

\section{Degenerate CR-immersions and Proofs of Theorems 2.2} and 2.1 .

This section is devoted to the proof of Theorem 2.2, where we assume that the CR-immersion $f: M \rightarrow \mathbb{S}$ has degeneracy $s \geq 1$.

Proof of Theorem 2.2. As in the proof of Theorem 2.1 in case $s=0$, we choose an admissible coframe $\left(\theta, \theta^{A}\right)$ on $Q$ near $f\left(p_{0}\right)$, adapted to $f(M)$, where $p_{0} \in M$ is now chosen such that the integer $s\left(p_{0}\right)$ defined in $(2.2)$ coincides with the degeneracy of $f$ (which is the minimum of $s(p)$ for all $p \in$ $M)$. As before, denote by $\left(\omega_{\alpha}{ }^{a} \beta\right)$ the second fundamental form of $f$ relative to this coframe. Since the mapping $f$ is constantly $\left(k_{0}, s_{0}\right)$-degenerate near $p_{0}$, by Proposition 7.1, the dimension of the span in (7.5) for $k=k_{0}$ is constant and equals to $d:=\hat{n}-n-s_{0}$ for $p$ near $p_{0}$. For the remainder of this proof, we shall use the indices $*$, \# running over the set $n+1, \ldots, n+d$ (possibly empty) and the indices $i, j$ running over $n+d+1, \ldots, \hat{n}$. Then 
after a unitary change of the $\left(\theta^{a}\right)$ if necessary, we may assume that

$$
\operatorname{span}\left\{\omega_{\gamma_{1}}{ }^{\#}{ }_{\gamma_{2} ; \gamma_{3}, \ldots, \gamma_{l}} L_{\#}, 2 \leq l \leq k_{0}\right\}=\operatorname{span}\left\{L_{\#}\right\}, \quad \omega_{\gamma_{1}}{ }^{j} \gamma_{2} ; \gamma_{3} \ldots \gamma_{l} \equiv 0, \quad l \geq 2 .
$$

We remark at this point that, if we can prove that the image $f(M)$ lies in a $(\hat{n}+1-s)$-dimensional plane $P$, then clearly the mapping $f: M \rightarrow \mathbb{S} \cap P$ is $k_{0}$-nondegenerate since the normal space of $M$ inside $\mathbb{S} \cap P$ would be $\cong \mathbb{C}^{d}$. We can write

$$
\hat{\omega}_{\#}^{j}=\hat{\omega}_{\#}{ }_{\mu} \theta^{\mu}+\hat{\omega}_{\#}^{j} \bar{\nu}^{\bar{\nu}}+\hat{\omega}_{\#}{ }^{j} \theta
$$

for suitable coefficients $\hat{\omega}_{\#}^{j} \mu, \hat{\omega}_{\#}^{j} \bar{\nu}$ and $\hat{\omega}_{\#}^{j}$. In view of the definition of the covariant derivatives (7.1-7.2), (9.1) immediately implies

$$
\omega_{\gamma_{1}}^{\#}{ }_{\gamma_{2} ; \gamma_{3}, \ldots, \gamma_{l}} \hat{\omega}^{j}{ }_{\mu}=0
$$

for any $l \geq 2$, and hence,

$$
\hat{\omega}_{\#}^{j}{ }_{\mu}=0 .
$$

Furthermore, in view of (3.19) and (4.3), (9.1) implies

$$
\hat{\varphi}_{\alpha}^{j}=\hat{D}_{\alpha}^{j} \theta, \quad \hat{\varphi}^{j}=\hat{D}_{\mu}^{j} \theta^{\mu}+\hat{E}^{j} \theta
$$

and

$$
\hat{\varphi}_{\alpha}^{\#}=\omega_{\alpha}^{\#}{ }_{\mu} \theta^{\mu}+\hat{D}_{\alpha}^{\#} \theta, \quad \hat{\varphi}^{\#}=\hat{D}_{\mu}^{\#} \theta^{\mu}+\hat{E}^{\#} \theta .
$$

Differentiating $\hat{\varphi}_{\alpha}^{j}$ and using the structure equations and the vanishing of the pseudoconformal curvature of $\mathbb{S}$, we obtain

$$
\omega_{\alpha}^{\#}{ }_{\mu} \theta^{\mu} \wedge \hat{\varphi}_{\#}^{j}=i\left(g_{\alpha \bar{\nu}} \hat{D}_{\mu}^{j}+g_{\mu \bar{\nu}} \hat{D}_{\alpha}^{j}\right) \theta^{\mu} \wedge \theta^{\bar{\nu}} \quad \bmod \theta
$$

By using (3.19) and (9.2) to compute $\hat{\varphi}_{\#}^{j}$ and identifying the coefficients of $\theta^{\mu} \wedge \theta^{\bar{\nu}}$, we conclude that

$$
\omega_{\alpha}^{\#}{ }_{\mu} \hat{\omega}^{j} \bar{\nu}=i\left(g_{\alpha \bar{\nu}} \hat{D}_{\mu}^{j}+g_{\mu \bar{\nu}} \hat{D}_{\alpha}^{j}\right) .
$$

Since the right hand side is conformally flat (see $\S 7$ ), \# runs over $n+$ $1, \ldots, n+d$, and $d=\hat{n}-n-s<n$ by the assumptions of Theorem 2.2, it follows from [Hu99, Lemma 3.2] (cf. e.g. the proof of Lemma 5.3) that

$$
\omega_{\alpha}^{\#}{ }_{\mu} \hat{\omega}_{\#}^{j} \bar{\nu}=0
$$

and, consequently, $\hat{D}_{\alpha}^{j}=0$. Thus, we have

$$
\hat{\varphi}_{\alpha}^{j}=0, \quad \hat{\varphi}^{j}=\hat{E}^{j} \theta .
$$


Substituting this information back into the structure equation for $d \hat{\varphi}_{\alpha}{ }^{j}$, using $d \hat{\varphi}_{\alpha}^{j}=0,(9.2),(9.5)$ and (3.19), and this time identifying terms involving $\theta^{\mu} \wedge \theta$, we conclude that

$$
\omega_{\alpha}^{\#}{ }_{\mu}\left(\hat{\omega}_{\#}^{j} 0+\hat{D}_{\#}^{j}\right)=0
$$

We claim that $\hat{\varphi}_{\#}^{j}=0$. To prove this it suffices to show

$$
\omega_{\gamma_{1}}^{\#}{ }_{\gamma_{2} ; \gamma_{3}, \ldots, \gamma_{l}} \hat{\omega}_{\#}^{j} \bar{\nu}=\omega_{\gamma_{1}}^{\#}{ }_{\gamma_{2} ; \gamma_{3}, \ldots, \gamma_{l}}\left(\hat{\omega}_{\#}^{j}{ }_{0}+\hat{D}_{\#}^{j}\right)=0, \quad l \geq 2 .
$$

We next differentiate (9.2) (with $\hat{\omega}_{\#}^{j} \mu=0$ by (9.4)), use (3.19) and the structure equation to compute $d \hat{\varphi}_{\#}^{j}$ modulo $\theta$ and identify the coefficients in front of $\theta^{\mu} \wedge \theta^{\bar{\nu}}$ to conclude that the covariant derivative $\hat{\omega}_{\#}^{j} \bar{\nu} ; \gamma$ is conformally flat. Here the covariant derivative is understood with respect to the induced pseudohermitian connection on the corresponding subspaces and quotient spaces, where the indices are running. Hence, all higher order covariant derivatives $\hat{\omega}_{\#}^{j} \bar{\nu} ; \gamma_{1}, \ldots, \gamma_{m}$ are also conformally flat (see $\S 7$ ). By taking covariant derivatives of (9.9) we obtain that the first expression in (9.12) is conformally flat. As before we conclude that it is actually zero by [Hu99, Lemma 3.2] since $d<n$. Similarly we obtain conformal flatness of the covariant derivative $\hat{\omega}_{\#}^{j} 0 ; \gamma$ by identifying the coefficients in front of $\theta^{\mu} \wedge \theta$ in the same identity. Again, all higher order derivatives are also conformally flat and we obtain the vanishing of the second expression in (9.12) by taking covariant derivatives of (9.11) and using [Hu99, Lemma 3.2]. This proves (9.12) and hence the claim $\hat{\varphi}_{\#}^{j}=0$ in view of (9.1). Finally, substituting this information back into the structure equation for $d \hat{\varphi}_{\alpha}^{j}=0$ and using (9.10), we conclude that $\hat{\varphi}^{j}=0$.

Now, we are in a position to complete the proof of Theorem 2.2. We have shown that $\hat{\varphi}_{\alpha}^{j}=\hat{\varphi}_{\#}^{j}=\hat{\varphi}^{j}=0$. As in $\S 8$ we realize the sphere $\mathbb{S}$ as the quadric $Q$ in $\mathbb{P}^{\hat{n}+1}$ and choose an adapted $Q$-frame $\left(Z_{\Lambda}\right)$ on $Q \cong \mathbb{S}$ near $f(p)$. We can further choose $\left(Z_{\Lambda}\right)$ satisfying $(8.5)$ with $\xi=0$ for our given admissible coframe $\left(\theta, \theta^{A}\right)$ as described in $\S 8$. It follows then from the second row in (8.5) and the symmetry relation $\hat{\varphi}_{A \bar{B}}=-\hat{\varphi}_{\bar{B}}$ that $\pi_{j}{ }^{\Omega}=0$ for all $\Omega$ except possibly $\Omega \in\{n+d+1, \ldots, \hat{n}\}$. Thus, we have

$$
d Z_{i}=\pi_{i}^{j} Z_{j}, \quad i, j \in\{n+d+1, \ldots, \hat{n}\}
$$

expressing the property that derivatives of the vectors $Z_{i}$ are linear combinations of $Z_{j}$ at every point. It follows that the span of $Z_{j}=Z_{j}(p)$ must be constant in the Grassmanian of $\mathbb{C}^{\hat{n}+2}$ for $p \in M$ near $p_{0}$. Hence the vectors $Z_{0}(p)$ lie in the constant $(\hat{n}+2-s)$-dimensional orthogonal subspace (with 
respect to the inner product (8.1)). Since, by definition of the adapted $Q$ frame, $Z_{0}(p)$ gives the reference point $p$ in $\mathbb{P}^{\hat{n}+1}$, we conclude that $f(p)$ is contained in a fixed $(\hat{n}+1-s)$-dimensional plane $P$ in $\mathbb{C}^{\hat{n}+1}$ for $p \in M$ near $p_{0}$. The minimality of $M$ implies now the inclusion $f(p) \in P$ for all $p \in P$ by the uniqueness of CR-functions. The last statement follows directly from the definitions. The proof of Theorem 2.2 is complete.

Proof of Theorem 2.1 in general case. Let $f: M \rightarrow \mathbb{S}$ be as in the theorem and denote by $s$ its degeneracy. By Theorem $2.2, f$ can be seen as a $k_{0^{-}}$ nondegenerate CR-immersion near some point of $M$ into a smaller sphere $\mathbb{S}^{\prime} \subset \mathbb{C}^{n+k-s+1}$. The degeneracy of the immersion obtained of $M$ into $\mathbb{S}^{\prime}$ is now 0 . The required conclusion follows now from the case $s=0$ considered in $\S 8$.

\section{References.}

[BER99] Baouendi, M.S.; Ebenfelt, P.; and Rothschild, L.P.: Real Submanifolds in Complex Space and Their Mappings. Princeton Math. Series 47, Princeton Univ. Press, 1999.

[CM74] Chern, S.-S.; Moser, J. K.: Real hypersurfaces in complex manifolds. Acta Math. 133, 219-271, (1974).

[CS83] Cima, J.; Suffridge, T. J.: A reflection principle with applications to proper holomorphic mappings. Math. Ann. 265, 489-500, (1983).

[CS90] Cima, J.; Suffridge, T. J.: Boundary behavior of rational proper maps. Duke Math. 60, 135-138, (1990).

[Fa86] Faran V, J. J.: On the linearity of proper maps between balls in the lower codimensional case. J. Differential Geom. 24, 15-17, (1986).

[Fa90] Faran V, J. J.: A reflection principle for proper holomorphic mappings and geometric invariants. Math. Z. 203, 363-377, (1990).

[F86] Forstnerič, F.: Proper holomorphic maps between balls. Duke Math. J. 53, 427-440, (1986).

[F89] Forstnerič, F.: Extending proper holomorphic mappings of positive codimension. Invent. Math. 95, 31-61, (1989). 
[Ho98] Hofer, H.: Dynamics, topology, and holomorphic curves. Proceedings of the International Congress of Mathematicians, Vol. I (Berlin, 1998). Doc. Math. 1998, Extra Vol. I, 255-280.

[Hu99] Huang, X.: On a linearity problem for proper holomorphic maps between balls in complex spaces of different dimensions. J. Differential Geom. 51, 13-33, (1999).

[KZ01] Kim, S.-Y.; Zaitsev, D.: The equivalence and the embedding problems for CR-structures of any codimension. Preprint (2001); http://arXiv.org/abs/math.CV/0108093.

[KN69] Kobayashi, S.; Nomizu, K.: Foundations of differential geometry. Vol. II. Interscience Tracts in Pure and Applied Mathematics, No. 15, Vol. II. Interscience Publishers John Wiley \& Sons, Inc., New York-London-Sydney, 1969.

[L01a] Lamel, B.: A reflection principle for real-analytic submanifolds of complex spaces. J. Geom. Anal. 11, no. 4, 625-631, (2001).

[L01b] Lamel, B.: Holomorphic maps of real submanifolds in complex spaces of different dimensions. Pacific J. Math. 201, no. 2, 357387, (2001).

[Le86] Lee, J. M.: The Fefferman metric and pseudohermitian invariants. Trans. Amer. Math. Soc. 296, 411-429, (1986).

[Le88] Lee, J. M.: Pseudo-Einstein structures on CR manifolds. Amer. J. Math. 110, 157-178, (1988).

[T75] Tanaka, N.: A differential geometric study on strongly pseudoconvex manifolds. Lectures in Mathematics, Department of Mathematics, Kyoto University, No. 9. Kinokuniya Book-Store Co., Ltd., Tokyo, 1975.

[W78] Webster, S. M.: Pseudohermitian structures on a real hypersurface. J. Differential Geom. 13, 25-41, (1978).

[W79] Webster, S. M.: The rigidity of C-R hypersurfaces in a sphere. Indiana Univ. Math. J. 28, 405-416, (1979).

P. Ebenfelt: Department of Mathematics

Universityof California at San Diego 
LA Jolla, CA 92093, USA

pebenfel@math.ucsd.edu

X. Huang: Department of Mathematics

RUTGERS UNIVERSITY

New Brunswick, NJ 08502, USA

huangx@math.rutgers . edu

D. Zaitsev: School of Mathematics

Trinity College

DUBLIN 2, IRELAND

zaitsev@maths.tcd.ie

ReCeived June 2002. 